\title{
Adjustable, short focal length permanent-magnet quadrupole based electron beam final focus system
}

\author{
J. K. Lim, ${ }^{*}$ P. Frigola, G. Travish, and J. B. Rosenzweig \\ Department of Physics and Astronomy, University of California at Los Angeles, Los Angeles, California 90095, USA \\ S. G. Anderson, W. J. Brown, J. S. Jacob, C. L. Robbins, and A. M. Tremaine \\ Lawrence Livermore National Laboratory, Livermore, California 94550, USA \\ (Received 8 March 2005; published 15 July 2005)
}

\begin{abstract}
Advanced high-brightness beam applications such as inverse-Compton scattering (ICS) depend on achieving of ultrasmall spot sizes in high current beams. Modern injectors and compressors enable the production of high-brightness beams having needed short bunch lengths and small emittances. Along with these beam properties comes the need to produce tighter foci, using stronger, shorter focal length optics. An approach to creating such strong focusing systems using high-field, small-bore permanent-magnet quadrupoles (PMQs) is reported here. A final-focus system employing three PMQs, each composed of 16 neodymium iron boride sectors in a Halbach geometry has been installed in the PLEIADES ICS experiment. The field gradient in these PMQs is $560 \mathrm{~T} / \mathrm{m}$, the highest ever reported in a magnetic optics system. As the magnets are of a fixed field strength, the focusing system is tuned by adjusting the position of the three magnets along the beam line axis, in analogy to familiar camera optics. This paper discusses the details of the focusing system, simulation, design, fabrication, and experimental procedure in creating ultrasmall beams at PLEIADES.
\end{abstract}

DOI: 10.1103/PhysRevSTAB.8.072401

PACS numbers: 41.85.Lc, 75.50.Ww, 41.50.+h

\section{INTRODUCTION}

In recent years, there has been a revolution in the design and implementation of electron sources due to the introduction of the rf photoinjector [1], which produces beams that have very high brightness, defined as simultaneous high peak current and low transverse emittance. The applications of high-brightness electron beams generated by such devices has also continued apace in this time. These applications, e.g., driving a plasma wakefield accelerator (PWFA) $[2,3]$ or providing an electron beam for an inverseCompton scattering (ICS) x-ray source [4,5], are strongly dependent on production of very dense beams. It has been widely noted that the scaling of such applications to higher performance levels (higher field in the PWFA, brighter for the ICS source) requires shorter bunch lengths $\sigma_{z}$ and lower transverse emittances $\varepsilon_{x, y}$, a situation that has strong implications for future development of electron beam sources themselves. These considerations have led to increased concentration on photoinjector optimization [6] and development of pulse compression systems. Much less emphasis has been placed, however, on the need to scale the dimensions of the beam focal systems down with other relevant length scales $-\sigma_{z}, \varepsilon_{x, y}$. The characteristic length scale of the focal system is the minimum " $\beta$ function," $\beta_{x}^{*}$, which is related to the transverse beam size at focus by $\sigma_{x}^{*}=\sqrt{\beta_{x}^{*} \varepsilon_{x}}$. In order to achieve a small transverse beam size at focus, a high-gradient quadrupole focusing array is required.

\footnotetext{
*Electronic address: jlim@ physics.ucla.edu
}

The difficulty in matching is due to two effects based in the method of preparing the beam: emittance compensation and pulse compression. When one compresses the electron beam, significant energy spread is introduced. In focusing the beam into the application interaction region, one must simultaneously avoid chromatic aberrations due to energy spread, and emittance growth due to residual beam space-charge effects. Assuming one uses relatively weak normal-conducting electromagnetic quads, to obtain a small focal spot, the beam must first be expanded for final focusing. In such a case, the final spot size, in the absence of an elaborate, long chromatically compensated finalfocus system, is limited by chromatic aberrations. These issues are illustrated by the following relation governing the ratio of final spot size $\sigma^{*}$ to initial $\sigma_{0}$ [7],

$$
\frac{\sigma^{*}}{\sigma_{0}}=\sqrt{\frac{1+\left(\beta_{0} / f\right)^{2}\left(\sigma_{\delta_{p}} / p\right)^{2}}{1+\left(\beta_{0} / f\right)^{2}\left[1+\left(\sigma_{\delta_{p}} / p\right)^{2}\right]}} \cong \frac{\sigma_{\delta_{p}}}{p},
$$

where the final term obtained in the limit $(\beta / f) \gg$ $\left(p / \sigma_{\delta_{p}}\right)$. When the initial $\beta$ function $\beta_{0}=\sigma_{0}^{2} / \varepsilon$ is larger than the effective focal length $f$ of the final-focus system, strong compression of the beam size is possible, up until the chromatic aberrations begin to dominate. There is a minimum in the beam size, for a fixed focal length system, when $\beta_{0} / f \cong p / \sigma_{\delta p}$. In this condition, the demagnification is $\sqrt{2} \sigma_{\delta p} / p$. With larger initial beam size, the chromatic contribution to the emittance grows rapidly and because of the demagnification limit, the final beam size actually grows with larger initial beam size. 
An example, which serves as the experimental context for the remainder of this work, serves to illustrate the implications of Eq. (1) on the choice of focusing scheme. A UCLA/LLNL collaboration has operated an ICS source experiment termed the picosecond laser-electron interaction for the dynamic evaluation of structures (PLEIADES) $[8,9]$, in which a $10-100 \mathrm{TW}$, sub-ps, $820 \mathrm{~nm}$ laser pulse is collided with a velocity compressed, sub-ps $50-75 \mathrm{MeV}$ electron beam. With the present generation of large aperture quads that UCLA employs, which have a maximum gradient of $15 \mathrm{~T} / \mathrm{m}$ over $\sim 10 \mathrm{~cm}$ length, the minimum value of the effective focal length of the final focusing triplet $f \approx 50 \mathrm{~cm}$, and thus optimum demagnification for $\sigma_{\delta p} / p=0.5 \%$ is only possible for $\beta_{0}=100 \mathrm{~m}$, or (with $\left.\varepsilon_{x}=\varepsilon_{n} / \gamma=0.14 \mathrm{~mm} \mathrm{mrad}\right) \sigma_{0}=3.2 \mathrm{~mm}$. We thus anticipate a minimum achievable spot size [Eq. (1)] of $\sigma^{*} \approx$ $32 \mu \mathrm{m}$. This spot-size estimate, which has been verified in simulation, is unacceptably large for the ICS source, which ultimately demands a factor of 4 smaller spot size for nonlinear ICS interaction studies, as well as for increased $\mathrm{x}$-ray photon production.

In addition, by allowing the beam to expand before final focus, space-charge forces (for beams of energy $<100 \mathrm{MeV}$ ) will increase the emittance [6], giving an even larger final spot size. This limitation also precludes more elaborate approaches, based on sextupole correction, to eliminate chromatic aberrations. At low energy therefore, transport and final focusing systems must be compact; short focal lengths are needed.

From this introduction, it is clear that one must look into the use of much shorter focal length lenses in order to create minimum $\beta$ functions small enough for many modern electron beam applications. One might consider superconducting quadrupoles or solenoids, but these devices are neither inexpensive nor easy to build with the necessary small (cm-scale) dimensions. On the other hand, permanent-magnet-based quadrupoles (PMQs) can access high-field gradients, and have been under study recently in two arenas: linear collider (LC) main accelerator focusing - to mitigate the cost of the needed power supplies and final-focus magnets in both circular accelerators [10] and LCs [11-14].

In the LC main accelerator application, an effort has been made to design a moderate strength, but tunable magnet [11]. Much of the international linear collider (ILC) oriented work has been concentrated on tunability, while simultaneously holding the magnetic center stable, as required for use in the beam-based alignment process. In addition, both ILC and CESR groups have studied the influence of their respective expected radiation environments on the magnetization of the permanent-magnet (PM) material [15], and found the effects to be small.

On the other hand, the need for extremely large field gradients makes the introduction of significant gradient tuning into a PMQ very challenging. In the present work, we discuss an alternative scheme that sidesteps these difficulties, by tuning the strength of the final-focus array (a class of quadrupole triplet) only through the longitudinal (z) positioning of the individual fixed-strength PMQs. In this way, the issue of tunability is separated from both that of alignment and that of achieving high gradient. Indeed, one may access extremely large field gradients by using an optimum, static intramagnet configuration. Such a magnet configuration was introduced by Halbach [16]. This magnet employs a pure permanent-magnet geometry consisting of 16 sections, with magnetization oriented at $0^{\circ}, \pm 45^{\circ}$, and $\pm 90^{\circ}$ with respect to their transverse symmetry plane. This type of magnet has been studied in the context of the compact linear collider (CLIC) program [12,13], and has been implemented at the Cornell Electron Storage Ring (CESR) in the CLEO detector mini- $\beta$ system [17].

The details of 3D field calculation in the Halbach sectioned PMQ geometry are discussed in the following section. This geometry is known to allow field gradients approaching the ratio $2 B_{r} / r_{i}$, where $B_{r}$ is the remanent field of the PM material, and $r_{i}$ is the pole inner radius. For the material used in the present study, $\mathrm{NdFeB}, B_{r}=$ $1.22 \mathrm{~T}$, and thus a $2.5 \mathrm{~mm}$ inner radius may produce over $600 \mathrm{~T} / \mathrm{m}$, a field gradient that is appropriate for use in the PLEIADES ICS experiment.

This can be seen from the following argument. Because the focal length of the system as a whole should be less than $10 \mathrm{~cm}$, the individual PMQ length is chosen to be $1 \mathrm{~cm}$. If one restricts, as is appropriate for a triplet configuration, the focusing phase advance (for the initial operating energy of $70 \mathrm{MeV}$ ) to at most one-half $\left[k_{q} l_{q}=\right.$ $\left.\left(B^{\prime} / B R\right) l_{q} \leq 0.5\right]$ in a single $1 \mathrm{~cm}$ lens, then over a $600 \mathrm{~T} / \mathrm{m}$ field gradient is deduced. We shall see below that this estimate on appropriate field gradient indeed yields effective guidelines for optimizing the beam optics in the PLEIADES final-focus system.

To allow an appreciation of the scope of challenges encountered in implementing such a tunable, ultrashort focal length final focusing array, we must present work on: magnet theory and design; beam dynamics simulation; fabrication, alignment and testing; and experimental beam measurements. In the following treatment, we therefore employ the following structure: the basic theory and simulation of a single PMQ magnet is discussed in Sec. II; the simulation of a focusing lattice is examined in Sec. III; Sec. IV is dedicated to the design and fabrication of the final-focus system; the measurement of the completed system is reviewed in Sec. V; and finally, implementation, usage, and beam measurements are presented in Sec. VI.

\section{MAGNET THEORY AND SIMULATIONS}

In this section, a 2D analytical model originally approached by Halbach and a 3D simulation model are presented to illustrate the dependence of the PMQ per- 
formance on the geometric and magnetic design parameters in the Halbach pure PM design.

We first discuss the two dimensional Halbach-type PMQ model, reviewing results that were originally established by Halbach. These results are then used to guide initial magnetic and beam optics design decisions.

To estimate the achievable gradients one obtains with a PMQ, we first assume a permanent-magnet geometry in which the magnetization varies continuously with an azimuthal angle $\phi$. The field gradient is given by

$$
B^{\prime}=2 B_{r}\left(\frac{1}{r_{i}}-\frac{1}{r_{o}}\right)
$$

With $B_{r}=1.22 \mathrm{~T}, r_{i}=2.5 \mathrm{~mm}$, and $r_{o}=7.5 \mathrm{~mm}$, we have $B^{\prime}=650 \mathrm{~T} / \mathrm{m}$. In practice, the field gradient is most strongly controlled by the inner radius $r_{i}$.

It is not possible to make a PMQ with a continuously varying remanent field, but the use of uniformly magnetized segments can well approximate the pure quadrupole case. This was recognized by Halbach, who studied the approximation to the ideal geometry that can be achieved by segmenting the magnet into $M$ geometrically identical pieces, which have magnetization rotated appropriately in each segment, as shown in Fig. 1. The segmentation of the magnet into $M$ sections diminishes the field gradient predicted in Eq. (2) by a factor $a_{2}$. For the Halbach geometry considered with 16 segments, the coefficient of quadrupole term, $a_{2}$, is found to be 0.94 - the field gradient is diminished by only $6 \%$ from the ideal geometry.

The model analysis above omits the effect of finite length of the PMQ. In order to address these effects, the magnetostatic simulation code RADIA [18] was used in 3D modeling and numerical analysis of a 16-piece Halbach PMQ. The magnetostatic object can be formulated as a

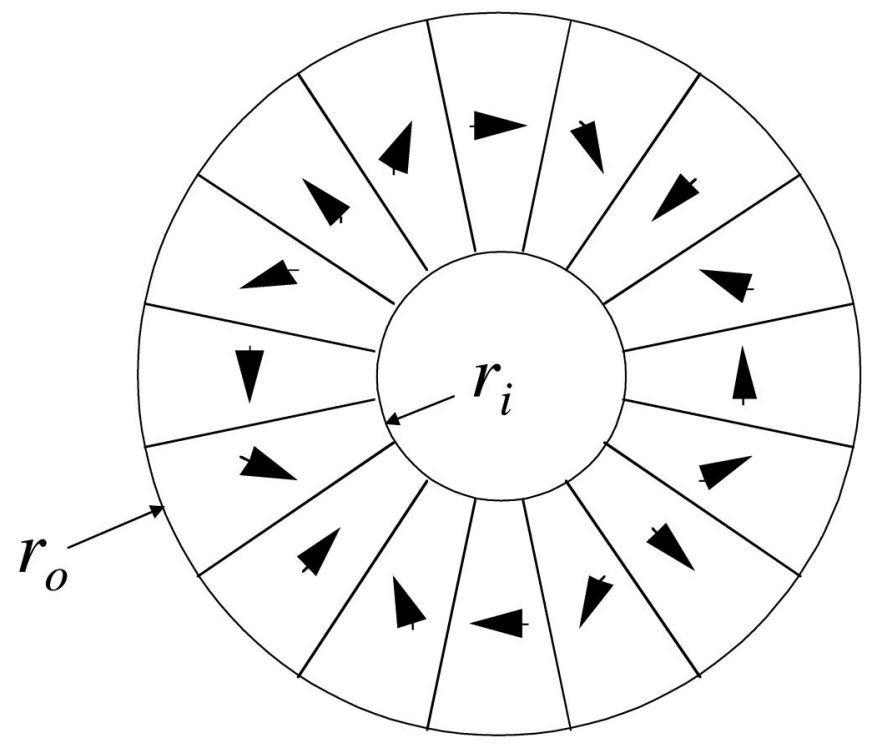

FIG. 1. A cross section of the 16 segment PMQ and the magnetization orientations shown as arrows. composition of a set of smaller geometric components bounded by planar polygons which then are linked to make up the desired magnet model. Furthermore, each component can be segmented into smaller subdivisions for improved accuracy.

The goal of our 3D simulation analysis is to obtain a set of optimized PMQ dimensions which, consistent with a given remanent field, yield an appropriately high-field gradient. Our application demands that the overall dimension of the PMQ be consistent with the beam optics dimensions (few $\mathrm{cm}$ focal lengths for each lens), and that the beam be passed through the small bore where the strong field gradient is produced. The design analysis also must factor in technical difficulties in the manufacturing process. $\mathrm{NdFeB}$, a rare-earth $\mathrm{PM}$ material, was chosen for our simulations over other rare-earth types, e.g., SmCo, because of our demand for a higher remanent field range.

Each component wedge block is bounded by two parallel trapezoidal planes separated by a length of $l_{z}$ and a given remanent field of $B_{r}$. By implementing a simple loop algorithm routine in RADIA, 16 wedge blocks are sequentially stacked side-by-side where the final model design, as seen in Fig. 1, is created. The final optimum PMQ dimensions giving compact geometry and desired field gradient determined from the simulations are $r_{i}=2.5 \mathrm{~mm}, r_{o}=$ $7.5 \mathrm{~mm}$, and $l_{z}=10.0 \mathrm{~mm}$. The field gradient that predicted from this parameter set is $B^{\prime}=573 \mathrm{~T} / \mathrm{m}$. Note that the gradient found in RADIA simulation is lower than that deduced from Eq. (2) mainly due 3D (edge) effects. The field gradient and the magnetic field profiles plotted in the bore region are shown in Fig. 2. The effective magnetic length determined from the simulation is $l_{\text {eff }}=10.4 \mathrm{~mm}$ (see Fig. 2).

We now discuss two of the most relevant factors contributing to changes in the performance of the $\mathrm{NdFeB}$ based PMQ: relaxation in PMQ due to intramagnetic interactions, and partial demagnetization due to temperature variations. The results of the demagnetization studies in RADIA are used to determine whether the coercivity and remanence in the $\mathrm{NdFeB}$ material are adequate.

The close proximity and high remanent fields of the segments can influence the overall magnetic characteristics of the assembled PMQs through mutual magnetic interactions. Relaxation modeling was performed by iterative calculations of the field strength and the magnetization of each wedge block under the influence of the other 15 wedge blocks, until the values reach the specified precision in the stabilized magnetization.

With the $\mathrm{NdFeB}$ magnet parameters from the material datasheet and the calculation precision set in RADIA, the simulation showed a negligible relaxation in the chosen geometry of the PMQ. However, a permanentmagnet material with a high coercivity is still desirable for its high resistance against opposing external fields and its resistance to temperature variation induced demagnetization. 

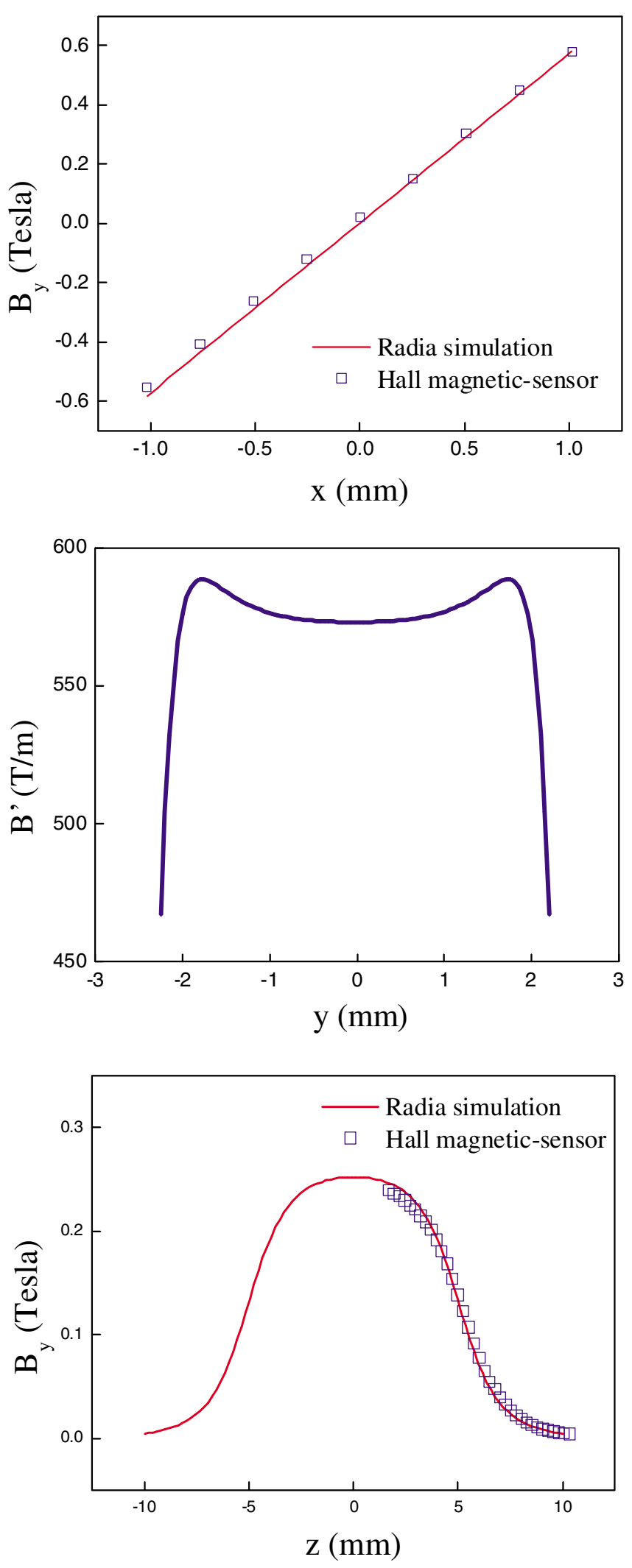

FIG. 2. (Color) 3D code RADIA PMQ field: a comparison of PMQ field profile measurements using a Hall probe to RADIA simulation results (top), the field gradient (middle), and the effective magnetic length taken at $r=2.0 \mathrm{~mm}$ (bottom).
Understanding the effect of temperature variation on the permanent magnet is important in the situation where an accidental exposure of a NdFeB PMQ to a temperature elevation can cause demagnetization as, for instance, during a vacuum bakeout. Demagnetization effects in the simulation studies are introduced through a second-order temperature dependence in the magnetization and the coercivity equations as [19]:

$$
\begin{array}{r}
M(T)=M\left(T_{0}\right)\left[1+a_{1}\left(T-T_{0}\right)+a_{2}\left(T-T_{0}\right)^{2}\right],(3 \mathrm{a}) \\
H_{c J}(T)=H_{c J}\left(T_{0}\right)\left[1+b_{1}\left(T-T_{0}\right)+b_{2}\left(T-T_{0}\right)^{2}\right],(3 \mathrm{~b})
\end{array}
$$

where $T_{0}$ is a reference temperature, and coefficients $a_{i}$ and $b_{i}$ are obtained from the material datasheet.

The relaxation of a PMQ magnetization is performed for 5 different chosen temperatures: $0,20,60,90$, and $120^{\circ} \mathrm{C}$. In Fig. 3, the irreversible loss of a PMQ magnetization is
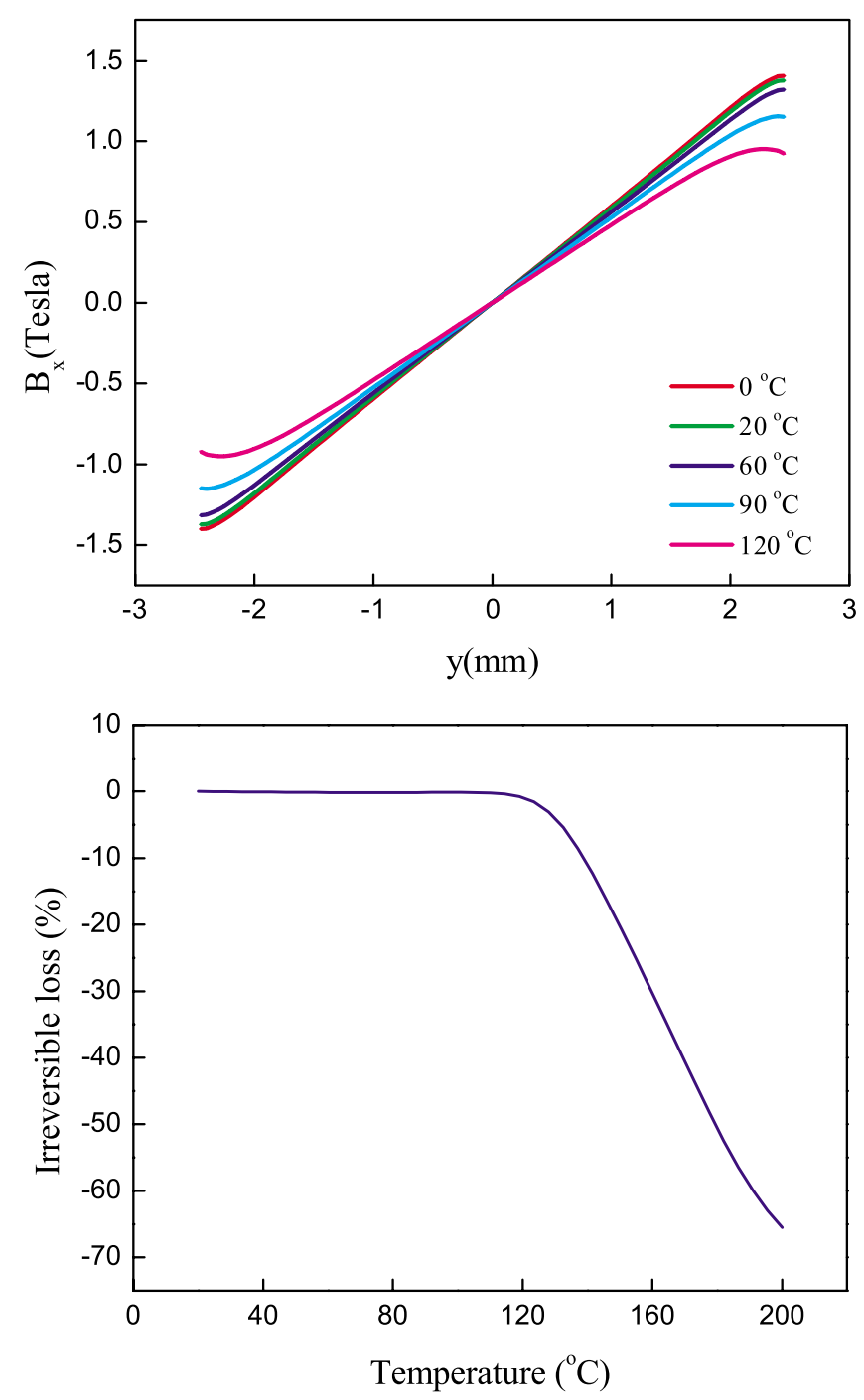

FIG. 3. (Color) Magnetic field spatial dependence showing decrease in slope as temperature value increases (top); percentage loss of field gradient as a function of temperature (bottom). 
shown as a function of a temperature change. This loss is small under $100{ }^{\circ} \mathrm{C}$, but it increases dramatically above $120^{\circ} \mathrm{C}$. This behavior is similar to that obtained from simulations reported in Ref. [19].

Geometric imperfections in PMQ due to fabrication and assembly errors, and their effect on magnet performance are investigated here in simulation. We concentrated our efforts in three causes of PMQ imperfections: angular orientation errors in the magnetic easy axis; sector-tosector geometric errors; and PMQ bore aperture radius errors. From simulations, approximate allowable tolerances in mechanical errors for the individual modules are obtained. A complete error budget, including alignment errors, is discussed in later sections.

In the first imperfection category, the following set of errors are introduced into the magnetization easy axes of randomly selected PMQ segments: $\pm 0.225^{\circ}, \pm 0.450^{\circ}$, $\pm 0.675^{\circ}$, and $\pm 1.125^{\circ}$, corresponding to absolute relative errors of $1 \%, 2 \%, 3 \%$, and $5 \%$, respectively. The magnetic field gradient is solved and presented for each of the angular error cases as shown in Fig. 4. As the magnitude of the angular error increases, the field profile suffers from a shift in the magnetic center and introduction of variation in the field gradient (higher-order multipoles) in the bore region.

For the second type of imperfection, the following errors in wedge angles (in this particular exercise, side length error is excluded, as we shall discuss in the magnet fabrication, that final boring out of inner pole surface yields a highly uniform surface) are chosen as the simulation input parameters: $\pm 0.225^{\circ}, \pm 0.450^{\circ}, \pm 0.675^{\circ}$, and $\pm 1.125^{\circ}$; again corresponding to relative errors of $1 \%, 2 \%, 3 \%$, and $5 \%$, respectively, as shown in Fig. 5. The error parameters are assigned to an arbitrary number of wedges in different positions which consequently create varying gaps. For the

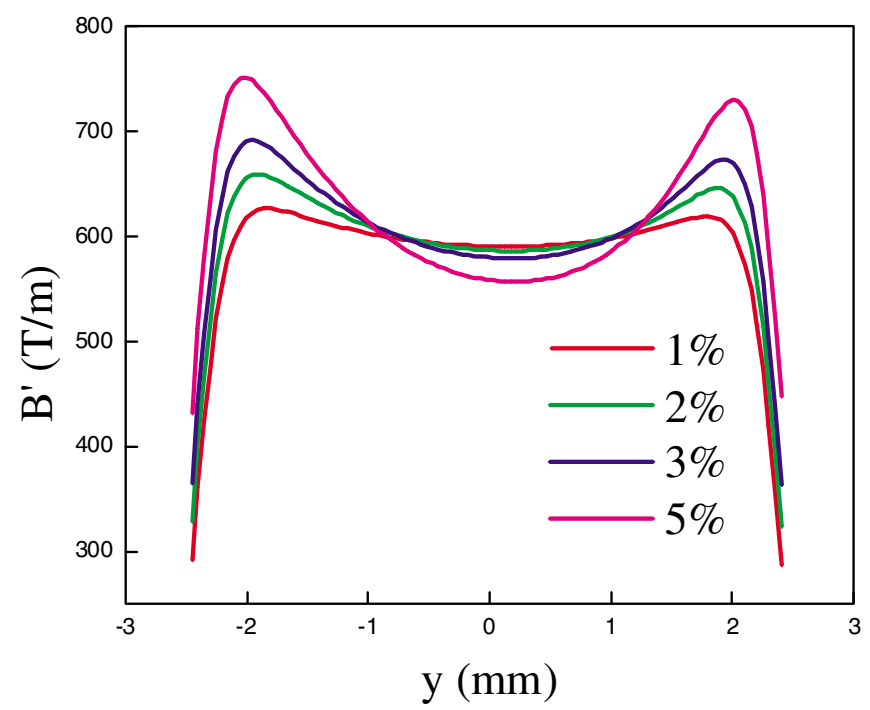

FIG. 4. (Color) Field gradient dependence on magnetization orientation errors.

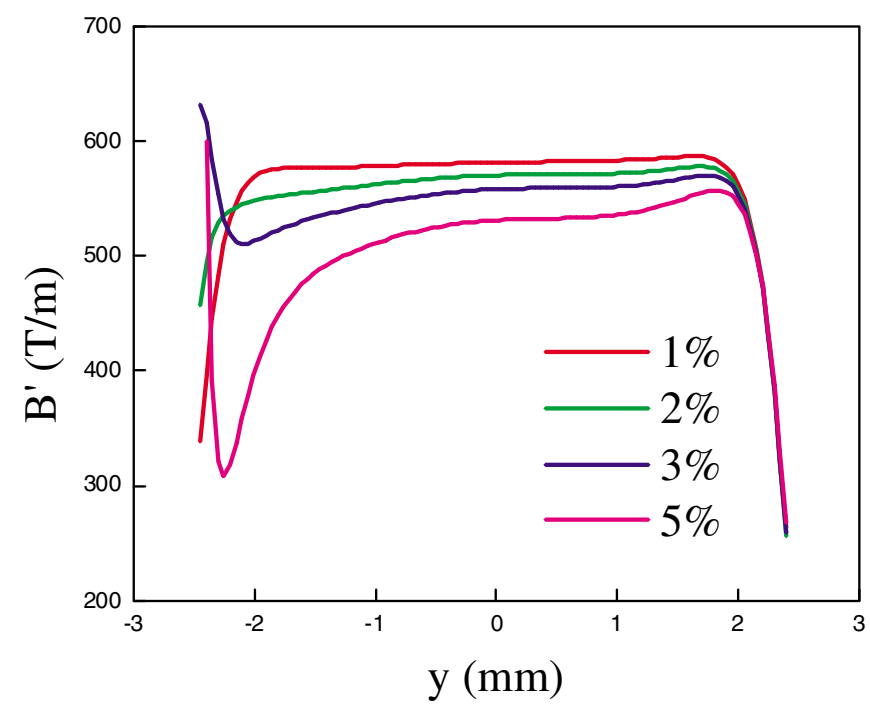

FIG. 5. (Color) Field gradient dependence on magnet wedge shape errors. Each curve represents wedge shape (mechanical) deviation from the ideal.

particular set of wedge angle errors introduced, the varying gap is tapered to the left side of the PMQ bore. The field gradient profile shows a significant sloping effect and a reduction in amplitude when the errors exceed $2 \%$.

As we shall discuss below, one of the final steps in the manufacturing process is boring out the PMQ hole to a specified value, in our case $2.5 \mathrm{~mm}$. In doing so, it is prudent to understand the effect of an undercut or overcut. As shown in the theoretical analysis, a variation in the PMQ bore radius is inversely proportional to the field. In the final case of our imperfect PMQ model, the following set of PMQ bore radii are considered: 2.45, 2.50, and $2.55 \mathrm{~mm}$ [Fig. 6]. As expected, the field gradient predicted

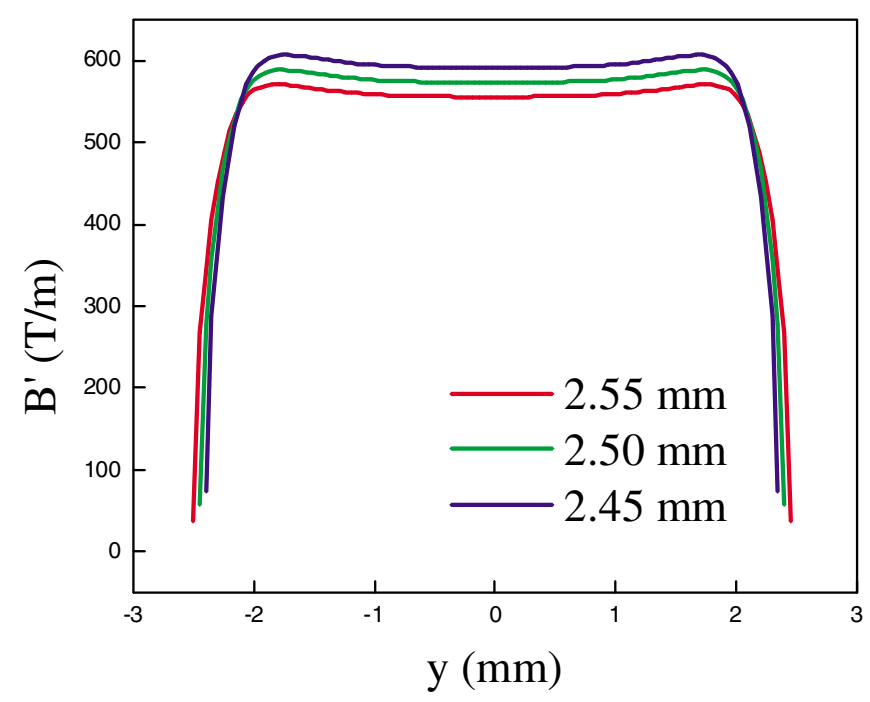

FIG. 6. (Color) Field dependence on magnet bore radius: $r=$ $2.45 \mathrm{~mm}$ (top), $2.50 \mathrm{~mm}$ (middle), and $2.55 \mathrm{~mm}$ (bottom). 
in different radius parameters gives 591 and $556 \mathrm{~T} / \mathrm{m}$, corresponding to an inner radius of 2.45 and $2.55 \mathrm{~mm}$, respectively. This variation is $\Delta B^{\prime} \approx \pm 18 \mathrm{~T} / \mathrm{m}$. In comparing with the 2D Halbach PMQ model, the variation is deduced from Eq. (2) to be $\left|2 B_{r}\left(\Delta r_{i} / r_{i}^{2}\right)\right| \approx 19.5 \mathrm{~T} / \mathrm{m}$.

The three notable error studies that have been performed provide the following tolerances which have been specified in our magnet design: (1) magnetization easy-axis angular orientation errors are allowed up to $2 \%$ before a notable change develops between the magnetic center and the mechanical center; (2) wedge shape (angle) error are allowed again up to $2 \%$; and, finally, (3) bore radius variations of $\pm 0.05 \mathrm{~mm}$ from the nominal $2.5 \mathrm{~mm}$ radius produce an acceptable field gradient variation of $\pm 3 \%$ from the ideal $B^{\prime}$. In Sec. III, magnet errors that are incorporated into the final-focus system have deleterious effects on the beam dynamics. The simulation of such effects are discussed next.

\section{BEAM DYNAMICS}

This section begins with the presentation on the theory of optimum triplet configuration based on thin-lens analysis. Then first-order beam simulations using the envelope code TRACE3D are employed to show the overall feasibility of the tunable final-focus system. Finally, with secondorder beam simulations performed with the multiparticle code ELEGANT, the effects of magnet errors and chromatic aberration effects on the final beam parameters are investigated.

\section{A. Optimized triplet design}

In this section the limitations on the final beam spot size using quadrupole triplet system are discussed and the optimum configuration of a simple triplet design is found. These design considerations motivate the choices of PMQ focusing system configuration as well as upstream matching optics.

Consider here an idealized system composed of three thin lenses separated by drift spaces and alternating between focusing and defocusing in the horizontal $(x)$ dimension. An illustration of this model problem is shown in Fig. 7. The assumed constraints for this design are that the focus be symmetric, and that the incoming beam is symmetric and at a waist, $\sigma_{x 0}=\sigma_{y 0}$ and $\sigma_{x 0}^{\prime}=\sigma_{y 0}^{\prime}=0$. The usual matrix formalism may be employed to describe the particle trajectories in the system. Using $M_{f}$ for a focusing thin lens (with a change in sign of $f$ in the case of a defocusing lens) and $M_{L}$ for a drift, the transport matrix of the system is simply $M=M_{L_{3}} M_{f_{3}} M_{L_{2}} M_{f_{2}} M_{L_{1}} M_{f_{1}}$.

For a given maximum obtainable focusing strength, or equivalently minimum focal length, $f_{\min }$, one seeks the parameters $f_{1}, f_{2}, f_{3}, L_{1}, L_{2}, L_{3}$ that maximize the convergence angle, $x^{\prime}$ at the focal point. Ignoring the small initial angle $\left(x_{0}^{\prime}=0\right)$, the final angle is given by $x_{f}^{\prime}=$

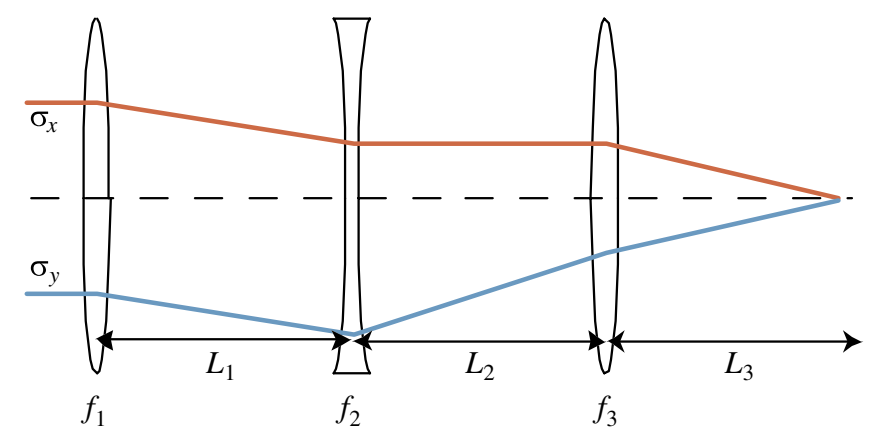

FIG. 7. (Color) Standard triplet design for a symmetric focus.

$M_{21} x_{0}$, where

$$
M_{21}=-\frac{1}{f_{3}}+\frac{1-\frac{L_{2}}{f_{3}}}{f_{2}}-\frac{1-\frac{L_{2}}{f_{3}}+L_{1}\left(-\frac{1}{f_{3}}+\frac{1-L_{2} /-f_{3}}{f_{2}}\right)}{f_{1}} .
$$

This expression can be simplified by requiring a round beam spot at the focus. This forces the matrix element $M_{21}$ to be equal for $x$ and $y$. Setting the right-hand side of Eq. (4) equal to its counterpart in $y$ leads to the relation:

$$
f_{1} f_{2}-f_{1} f_{3}+f_{2} f_{3}-L_{1} L_{2}=0
$$

Similarly, requiring $x=y$ at the third lens by equating the $\tilde{M}_{11}$ elements of the $x$ and $y$ versions of the matrix $\tilde{M}=$ $M_{L_{2}} M_{f_{2}} M_{L_{1}} M_{f_{1}}$ gives the equation

$$
f_{1}=f_{2}\left(1+\frac{L_{1}}{L_{2}}\right)
$$

The expected result shown by Eq. (6) is that the second lens must be stronger than the first, provided $L_{1}>0$.

Applying Eqs. (5) and (6) to Eq. (4) and solving for variables $f_{2}, L_{1}$, and $L_{2}$ leads to

$$
M_{21}=-\frac{1}{f_{2}^{2}} \frac{L_{1} L_{2}}{L_{1}+L_{2}} .
$$

Now, if it is assumed that the second lens provides the shortest focal length, $f_{2}=f_{\min }$, the minimum spot size is achieved by maximizing the expression $L_{1} L_{2} /\left(L_{1}+L_{2}\right)$, subject to the constraints given by Eqs. (5) and (6) and the fact that $f_{3} \geq f_{\min }$. These three conditions lead to the inequality, $L_{1} L_{2} \leq f_{\min }^{2}$, which is sufficient to find that $L_{1} L_{2} /\left(L_{1}+L_{2}\right)$ is maximized when $L_{1}=L_{2}=f_{\min }$. With back substitution, the optimum triplet configuration has the following parameters:

$$
L_{1}=L_{2}=L_{3}=f_{2}=f_{3}=f_{\min } \quad \text { and } \quad f_{1}=2 f_{\min } .
$$

Note that if Eqs. (4)-(6) were solved for $f_{3}$ and the assumption $f_{3}=f_{\min }$ is made, the maximum convergence angle still occurs with the parameters of Eq. (8), showing this (a $2 f,-f,+f$ asymmetric triplet lens configuration) 
to be the optimal design. We have adopted this design philosophy in our PMQ-based final-focus system.

\section{B. Simulations using TRACE3D}

We employ the quad triplet configuration suggested by the thin-lens analysis of the previous section, compactly indicated by F-DD-FF. The double strength (half-focal length) lenses are created by concatenating two identical magnets of the type discussed in Sec. II. The double-length magnets are moved in tandem, to act as a movable single lens. This asymmetric triplet is first studied using TRACE3D [20], an envelope code based on a first-order matrix description of the transport. The optimum lattice configuration was studied with this code to establish tuning conditions and performance predictions across a wide range of beam energies. Further, as is discussed below, the code is employed as a tuning guide in the experimental application of the focusing system.

TRACE3D studies demonstrated the focusability of a wide range of beam energies by dynamically configuring the drift spaces between PMQ lenses. Two examples of such simulation studies are illustrated in Fig. 8 for two different beam energy settings: 72 and $92 \mathrm{MeV}$. The following beam parameters (informed by upstream measurements at PLEIADES) served as input: rms emittances of $\varepsilon_{x}=$ $7 \mathrm{~mm} \mathrm{mrad}, \varepsilon_{y}=15 \mathrm{~mm}$ mrad and $\beta$ functions of $\beta_{x}=$ $2.0 \mathrm{~m}, \beta_{y}=0.9 \mathrm{~m}$. TRACE3D results, shown in Fig. 8 , illustrate that the magnet spacings $L_{1}, L_{2}$, and $L_{3}$ between quadrupole magnets expand with increasing electron beam energy, as expected, in order to increase the focal power of the triplet as it is applied to a more rigid beam.

Lower beam energy operation, below $60 \mathrm{MeV}$, is not possible in a system where $560 \mathrm{~T} / \mathrm{m}$ PMQs are utilized. To access this lower energy range one may employ a weaker focusing lens configuration. The following system has been recently implemented for lower energy (20$35 \mathrm{MeV}$ ) ICS experiment mode: weak focus - strong defocus - strong focus (WF-SD-SF). Here the weak PMQ is created by boring out an existing PMQ to a larger inner radius, which produces a measured field gradient of
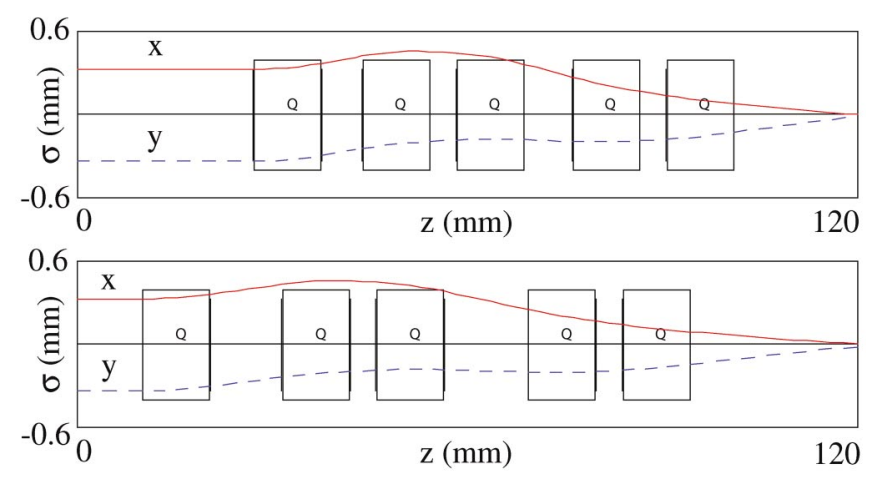

FIG. 8. (Color) Beam energy: $72 \mathrm{MeV}$ (top) and $92 \mathrm{MeV}$ (bottom).
$270 \mathrm{~T} / \mathrm{m}$. In addition to replacing the first PMQ with a weaker PMQ, the two following lenses consist of a single PMQ as opposed to a double PMQ in the original configuration. TRACE3D simulation of this weaker focusing array which show system tunability down to $30 \mathrm{MeV}$, as has been recently verified experimentally.

Detailed multiparticle simulation studies, performed with a sophisticated higher-order matrix calculation code, ELEGANT, of effects arising from the presence of errors on the performance of the PMQ final-focus system are presented in the following section.

\section{Simulations using ELEGANT}

There are several types of intratriplet alignment errors which may have profound effects upon the performance of the final-focus system. The two most serious are rotated (skew) PMQs and transverse magnetic center offset error. A third source of error not addressed in the previous section is the effect of chromatic aberrations.

Understanding the effects of these errors requires use of a more powerful tool for analysis, the higher-order envelope and tracking code ELEGANT [21]. The effects of these errors are examined here separately. Solutions for mitigating the effects of the mechanical errors in the final-focus system's overall design are discussed below which effectively correct errors to within tolerances allowed.

A systematically rotated field in a quadrupole magnet is expected when the 4-fold symmetry of the quadrupole is broken by an error in manufacturing process, for instance of uneven wedge sector shapes and incorrectly magnetized blocks. In addition, errors in angular placement of the PMQs with respect to the beam line may also produce a skewed magnet.

In the simulation code, the skew rotation term is individually introduced in each quadrupole. In our study, we outline a tolerance envelope in rotation by applying a systematic error. A clockwise rotation error is applied to focusing quadrupoles and a counterclockwise rotation error applied to defocusing quadrupoles. Based on the simplified model constructed, ELEGANT simulations are generated for three different cases of magnet rotation angle errors: $0,0.01$, and 0.02 radians. The normalized emittances in both transverse dimensions display growth proportional to the rotation error, as shown in Fig. 9. The final rms beam spot size grows from 20 to $34 \mu \mathrm{m}$ when a $0.01 \mathrm{rad}$ systematic rotation error was introduced. The beam spot size produced of course grows progressively worse with increasing rotation; in the case of 0.02 rad rotation error, a $200 \%$ growth in final beam spot size is found.

In actuality, all of the PMQs shared the same magnet characteristics, as they are all created by slicing each magnet from a long assembly. Thus, a rotation error due to a segment or magnetization variability found in one particular magnet is found also in the other magnets and therefore an azimuthal rotation error is essentially elimi- 


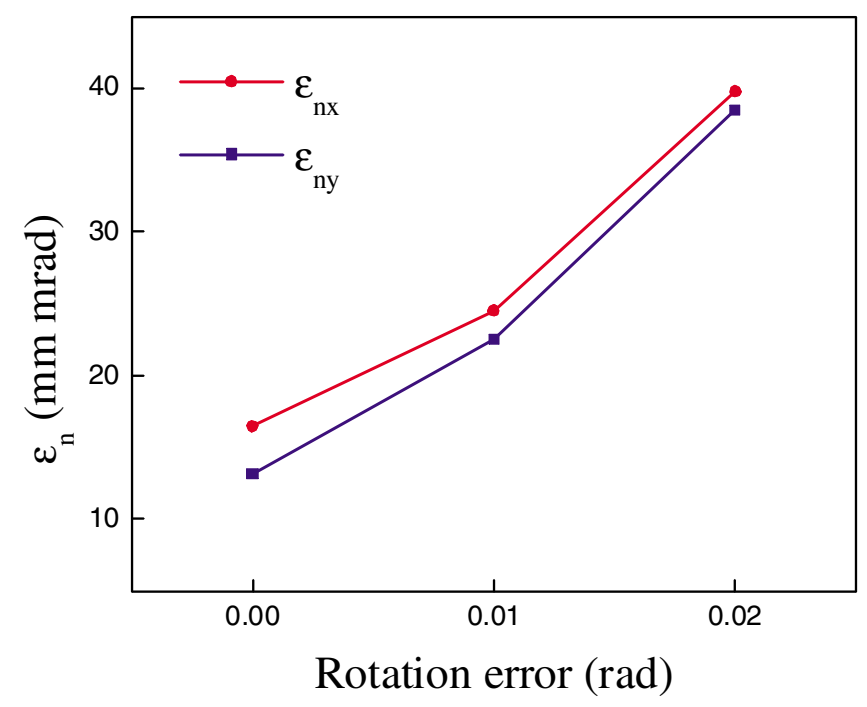

FIG. 9. (Color) Rotation error effect on normalized emittance growth through an otherwise ideal triplet, as simulated by ELEGANT.

nated from the focusing system. Any other rotation errors can be also introduced through incorrect mounting of the lens assembly. Therefore, considerable emphasis was placed on mechanical tolerances. In particular, magnet holders for enclosing PMQs are specifically machined to comply with less than $0.01 \mathrm{rad}$ angular error.

As noted in the magnet simulations of magnet easy-axis orientation and wedge errors, the magnetic center is shifted from the nominal geometric center of PMQ due to the cumulative effect of magnet errors. When such PMQs are installed into the translation rail of the final-focus system, magnet centers of the focusing and the defocusing PMQs will be in offset positions due to $90^{\circ}$ rotation relation. The effect of quad misalignments is to produce steering; if the vertical midplane of a quadrupole is displaced vertically by $\delta y$, it will introduce a dipole field $\delta B_{x}=B^{\prime} \delta y$. Furthermore, this steering produces momentum dispersion, which also leads to degraded performance. Determining the acceptable tolerance in magnet displacement places demands on the types of adjustments one needs in mechanical design of magnet holders. In practice, with magnetic center measurement data, corrections are applied through adjustments, after centerline measurement of each magnet, of the PMQ holder.

To simulate misalignments, the PMQs are simply specified to have transverse misalignments in ELEGANT. The focusing PMQs are assigned a vertical displacement error, while the defocusing PMQs the same horizontal displacement. The following values in displacement error are used: $0,0.1,0.2$, and $0.4 \mathrm{~mm}$. The displacement error's effect on the horizontal normalized emittance is less evident when compared to the vertical emittance as the beam encounters a greater maximum misalignment in this direction, as shown in Fig. 10. The emittance growth due to misalign-

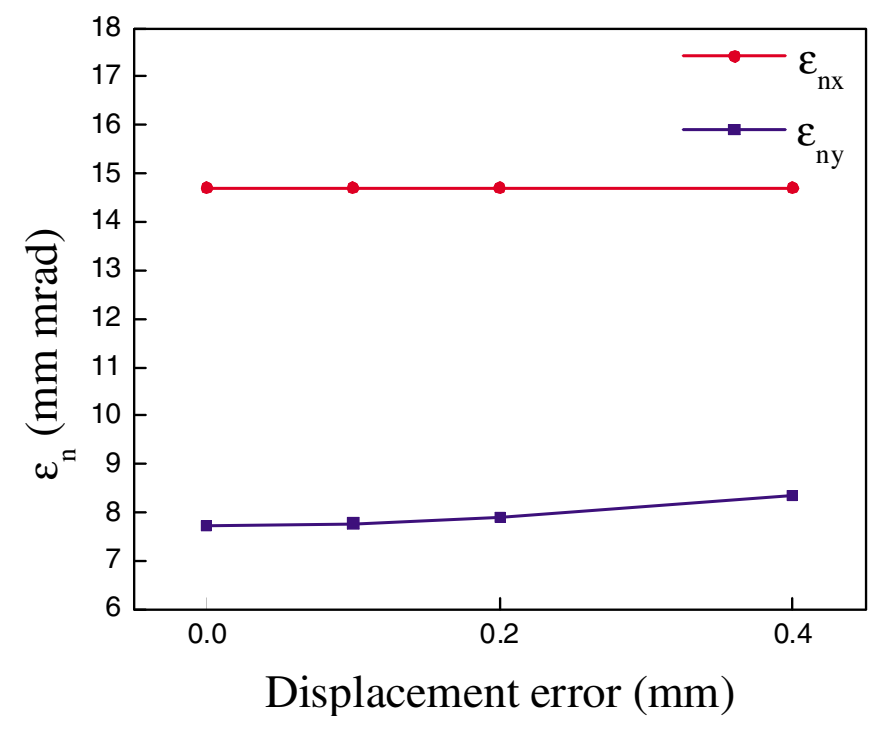

FIG. 10. (Color) Effect of magnet-center errors on emittance.

ment is less severe than that due to the skew rotation error, because it is driven mainly by momentum dispersion, which is a small effect for the design $\sigma_{\delta p / p}=0.5 \%$. The vertical emittance growth is near negligible up to $0.1 \mathrm{~mm}$ misalignment, growing to $8 \%$ at $0.4 \mathrm{~mm}$ displacement from the centers.

As noted in the introduction, the use of short focal length magnets in the final-focus system is in fact motivated by the need to evade chromatic effects on our ICS x-ray experiment at PLEIADES. The focusing error from chromatic aberration causes a severe case of a beam demagnification, which creates a low dense electron beam at the interaction, and hence low ICS $\mathrm{x}$ rays are generated. The effects of beam momentum spread effect on the final focused beam spot have been simulated with ELEGANT, and are shown in Fig. 11.

As mentioned in the introduction, chromatic aberration becomes the dominant factor contributing to the final beam size as the input beam size $\sigma_{0}$ is expanded. Since Eq. (1) was derived as a generalization to a thick triplet lens, as opposed to the original first-order, thin-lens treatment found in Ref. [7], a simulation study of its validity was performed using ELEGANT. The results of this analysis are plotted in Fig. 12. In this study, we used the original design emittance of the PLEIADES injector, so the overall scale of the achievable spot sizes is smaller.

As can be seen in Fig. 12, chromatic aberration dominates the initial beam emittance when the beam at finalfocus entrance $\sigma_{0}$ exceeds the optimum size,

$$
\left.\sigma_{0}\right|_{\mathrm{opt}}=\sqrt{\frac{\varepsilon_{x 0}}{\sigma_{\delta_{p}} / p} f} .
$$

The corresponding minimum final beam spot size obtained 

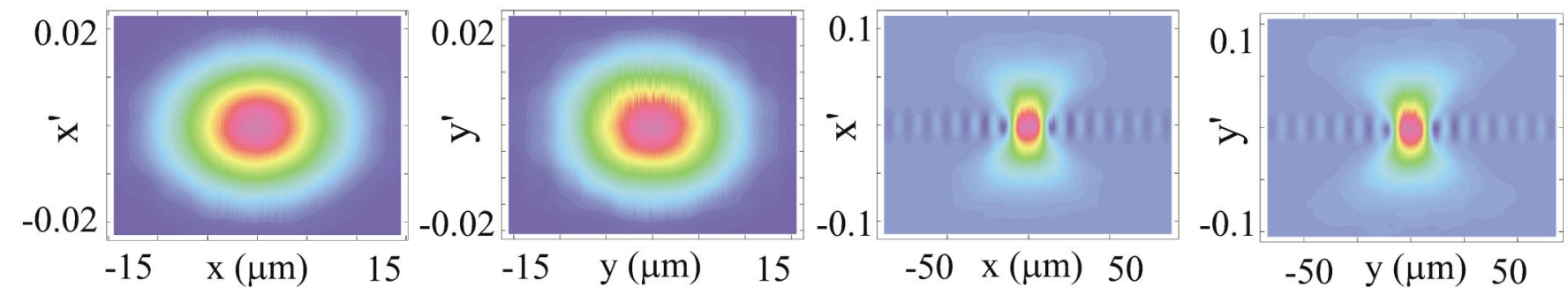

FIG. 11. (Color) ELEGANT simulation of chromatic effects: $x$ and $y$ phase space plots at the focal point; $\sigma_{\delta p / p}=0$ case (1st and 2nd) and $\sigma_{\delta p / p}=0.5 \%$, showing chromatic aberrations (3rd and 4th).

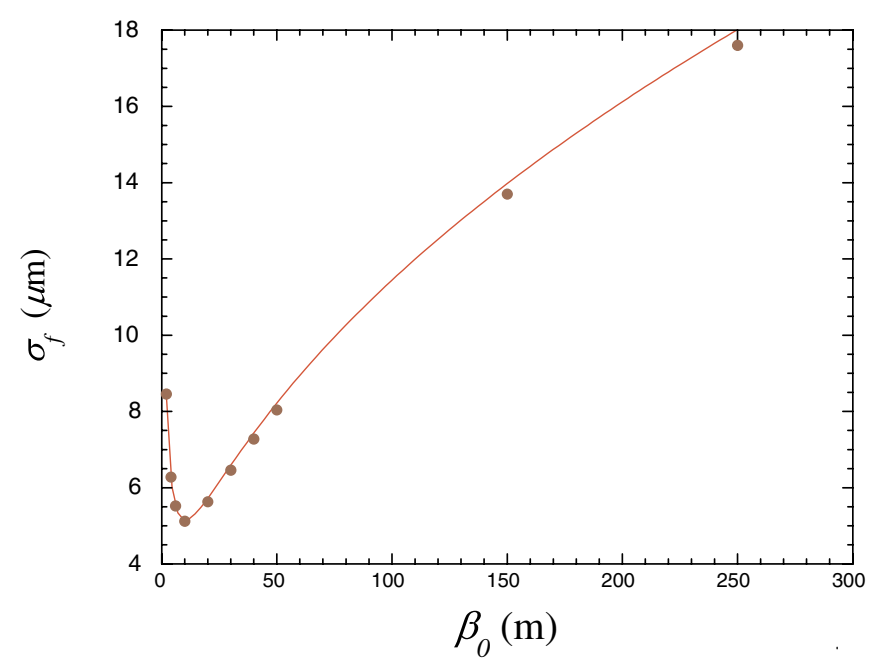

FIG. 12. (Color) Study of chromatic aberration effects in PLEIADES PMQ final-focus system, using ELEGANT simulation (dots), with theoretical prediction (line).

in the presence of the chromatic aberrations is

$$
\left.\sigma^{*}\right|_{\min }=\sqrt{2 f} \sqrt{\varepsilon_{x 0} \frac{\bar{\sigma}_{\delta_{p}}}{p} .}
$$

The simulations shown in Fig. 12 assume a round input beam with initial $\beta$ functions in the range $2 \mathrm{~m} \leq \beta \leq$ $250 \mathrm{~m}, 0.6 \%$ fractional rms momentum spread and $3.6 \times$ $10^{-2} \mathrm{~mm}$ mrad rms geometric emittances. The ELEGANT results are plotted against the prediction of Eq. (1). In this way, the effective focal length of the final-focus system in the $x$ dimension was determined to be $f_{\text {eff }}=6.2 \mathrm{~cm}$. The predicted optimum input $\beta$ function for the minimum final beam focal spot size of $5 \mu \mathrm{m}$ is $10 \mathrm{~m}$ for this case, which has a relatively small emittance. For the actual experimental parameters with degraded emittance, the initial optimum $\beta$ is smaller.

\section{SYSTEM DESIGN AND PROPERTIES}

\section{A. Magnet design and fabrication}

Fabrication of an ultrahigh field gradient Halbach-type PMQ with good field quality has proven to be challenging from an engineering perspective. Assembling highly magnetized, small trapezoidal segments into a precisely aligned unit with a small bore poses many mechanical challenges. In addition, fabrication of multiple PMQs with both identical magnetic and geometric characteristics presents further difficulties.

Nevertheless, a simplified fabrication procedure for producing a high quality, and ultrahigh gradient PMQ may be given as follows. A long trapezoidal wedge which is premagnetized to a $B_{r}=1.22 \mathrm{~T}$ is machined to a specified design shape from a larger magnet block through a high precision cutting process, wire electrical discharge machining (EDM). The 16 magnetized wedges are then coupled together carefully into an equally long aluminum keeper tube. In addition to magnet wedges being held inside the keeper, a thin layer of cohesion material is applied, strongly joining neighboring magnets. The tube keeper and a bonding glue together ensure structural stability of the PMQ under extreme magnetic repulsions. The long assembly is then cut into six identical PMQs, with a mechanical length of $10 \mathrm{~mm}$, to ensure consistent performance between all PMQs. In particular, construction of a single, long magnet assembly assured that the relative azimuthal (roll) errors of individual units may be essentially eliminated. Finally, the inner diameter of bore is slowly ground to the specified value of $5 \mathrm{~mm}$ with a diamond cutter. The PMQ fabrication procedure is shown schematically in Fig. 13.

The manufacturing process required uniform magnet material properties because no sorting process was relied upon when selecting for the individual magnet wedges.

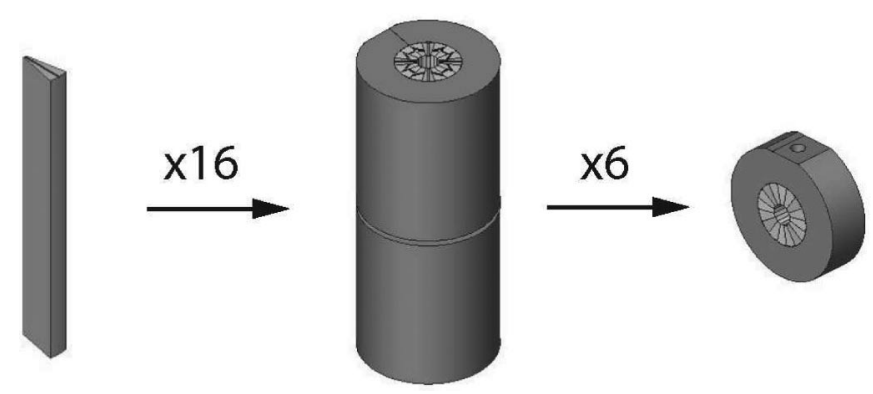

FIG. 13. An illustration of the PMQ manufacturing process. 
Instead, the long magnet wedges were all machined from a single premagnetized block, with the field orientation assured by the cut angle rather than a post-machining magnetization. This recipe transferred the possible sources of error and precision to the machining process, and spared us from having to measure very small magnets and sort hundreds of wedges.

For the application at hand, a particular grade of neodymium iron boride $(\mathrm{NdFeB})$ was selected based on various parameters, availability, and homogeneity. Key parameters of the $\mathrm{NdFeB}$ (grade $35 \mathrm{SH}$ ) used in the PMQs are listed in Ref. [22].

\section{B. Mover system}

Before implementing the PMQ system on PLEIADES, general problems associated with the application of this focusing system in the experimental environment had to be solved. Addressing the mechanical tolerances and space constraints of the PMQ system, while maintaining an environment consistent with ultrahigh vacuum (UHV) and magnetic fields, required careful integration.

Precision machining of all parts eliminated the need for adjustable alignment in the movers. Computer controlled custom vacuum linear motion feedthroughs were utilized to control each quadrupole's longitudinal position, which was precisely constrained by a rail system. Motion of the PMQs was set by use of stepper motors and LabVIEW control software. The entire magnet axis of the system was aligned by use of a pulsed-wire system and optical techniques. Other features of the assembly include a ceramic ablation disc at the front of the assembly to protect the magnets from misaligned high-intensity laser damage (during the ICS experiment), and a scintillating disc at the (beam entrance) side of the assembly, for locating mis-steered electron beams.

From a mechanical design approach, the system had to meet the following requirements: independent (preinstallation) correction of magnetic center to mechanical center errors for each PMQ assembly, independent position control of PMQs to within $100 \mu \mathrm{m}$ in the beam line direction, magnetic center-to-center transverse placement with $10 \mu \mathrm{m}$ precision for each PMQ along the range of motion, insertion of assembly into a standard $4^{\prime \prime}$ inner diameter (ID) vacuum chamber, and compatibility with vacuum at a nominal pressure of $<10^{-6}$ Torr.

A mechanically simple design was chosen to comply with these stringent requirements. A precision-machined stainless steel Conflat flange was fabricated to serve as a reference monument, mechanical support, and vacuum seal. Three precision ground rods were fitted into the flange by means of expansion and shrinkage fits.

Pairs of magnets were supported by precision-machined aluminum disks, each with three linear bearings press fit into the outer diameter. The disks and bearings move along the rods, and are controlled by vacuum linear motion

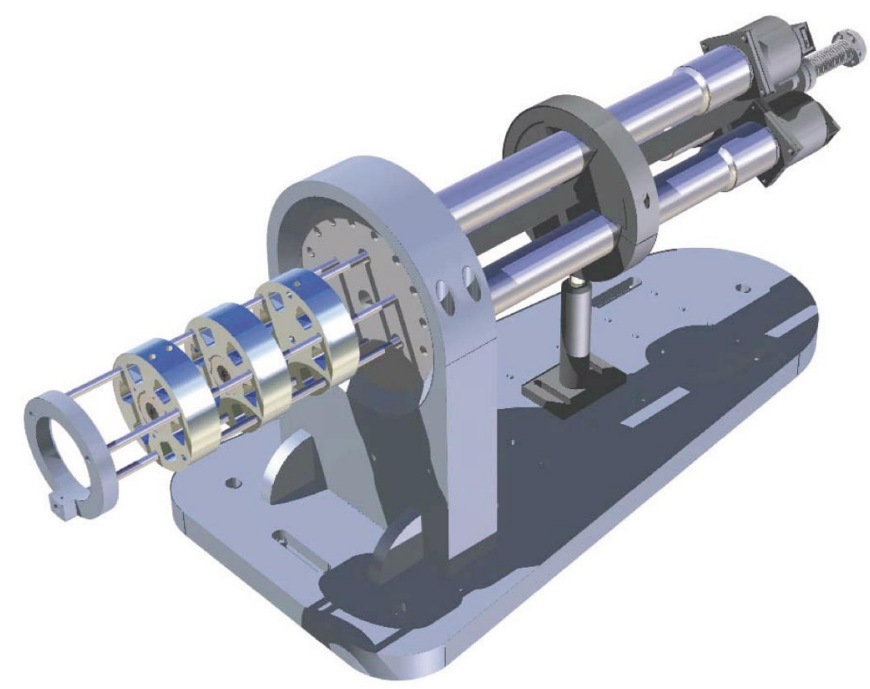

FIG. 14. (Color) Rendered CAD drawing of the final-focus assembly.

feedthroughs. The entire mechanical assembly is supported by the main flange using a massive precision ground stand, which affords five-axis alignment, as shown in Fig. 14.

An initial set of disks were machined to place each PMQ into the mechanical center of the assembly $\left( \pm 0.0005^{\prime \prime}\right)$. Using optical techniques along with a pulsed-wire system, the magnetic center of each PMQ was found relative to the assembled mechanical centers. New discs, if needed, were then machined with appropriate offsets to eliminate these errors. Fiducials were placed inside the front and back PMQ for optical alignment.

\section{PMQ SYSTEM MEASUREMENTS}

Various measurements on the PMQs were performed using pulsed wire and Hall probe. In comparison with the 3D magnetic simulations and tolerance studies, the quadrupole magnet properties indeed agree well with predicted values. More details of measurement techniques are discussed in the following sections.

Measurements of the PMQs were made with the pulsedwire technique [23]. The test setup included a single meterlong, $50 \mu \mathrm{m}$ diameter $\mathrm{BeCu}$ wire which was stretched out through the PMQ magnetic field region and anchored at end mounts. One end was fixed, while the other end had a pulley over which the wire was tensioned by a hanging weight. A pulse of $20-50 \mathrm{~V}$, with a duration of a few msec to $\mathrm{sec}$, and a repetition rate of $1 \mathrm{~Hz}$ was produced from a high voltage pulser triggered by a function generator. When the current pulse is passed along the wire through the PMQ magnetic region, it interacts with the PMQ $B$ field and deflects the wire. A detector consisting of a photodiode sensor and a diode laser was employed to quantify the deflection. Two sets of laser-sensors were used in order to measure both transverse deflections simultaneously. 
The pulsed-wire technique was used to detect nonlinearity in the $B$-field dependence on transverse displacement. The $\mathrm{BeCu}$ wire was translated horizontally through the magnetic center from -2.0 to $+2.0 \mathrm{~mm}$. The deflection amplitude gives a measure of the (longitudinally) integrated vertical component of the $B$ field. According to the data reported in Fig. 16, the transverse $B$ field shows no discernible higher multipole content; the dependence of $B_{y}$ on $x$ is linear to within measurement error over the scan interval.

The PMQ magnetic center to mechanical center alignment has also been studied using the pulsed-wire technique. A PMQ mounted on $2 \mu \mathrm{m}$ stages, with the wire initially located at the magnet mechanical center, is continuously adjusted in transverse position until the magnetic center is found by observing that the wire deflection signal is reduced to near zero (see Fig. 15). From this measurement, it was determined that the magnetic center was aligned to the geometric center to within the instrument resolution of $25 \mu \mathrm{m}$.

The alignment of the final mover system was observed with a theodolite for horizontal motion and an autolevel for vertical motion. The alignment in the horizontal direction was good to within $25 \mu \mathrm{m}$. The autolevel indicated notable sagging in the vertical direction, however. The sagging was compensated by a set screw located on the bottom of the ring piece which ties together the free ends of the rails.

Field gradient measurements of the PMQ were attempted using the pulsed-wire technique. However, the wavelength dependence of the wire response prevented calibration of the $1 \mathrm{~cm}$ long magnet measurements, as this was attempted using the known fields in a much longer $(10 \mathrm{~cm})$ electromagnetic quadrupole. As an alternative option for quantitative measurements, a miniature magnetic Hall probe was employed to determine the field gradient and the effective magnetic length of the PMQ.

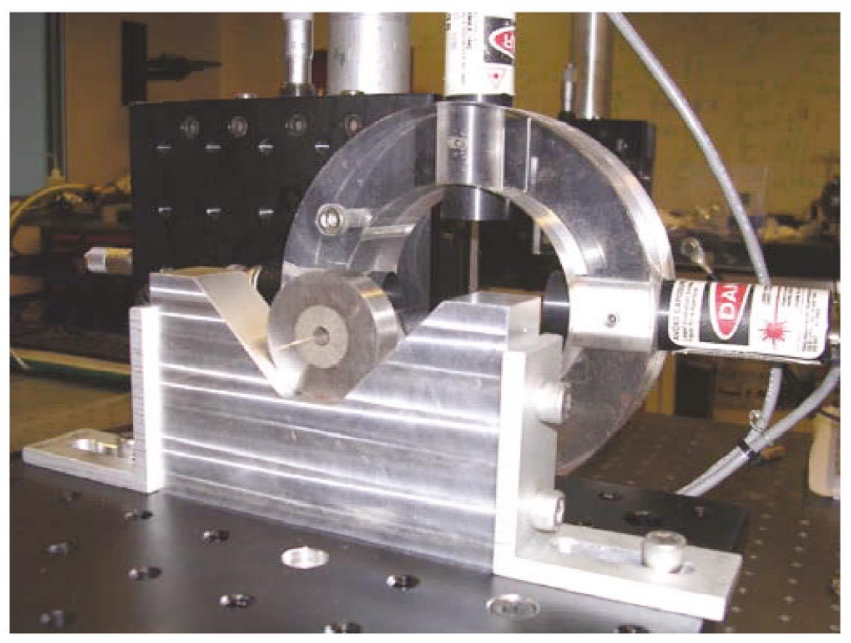

FIG. 15. (Color) Single PMQ module on the pulsed-wire measurement system.

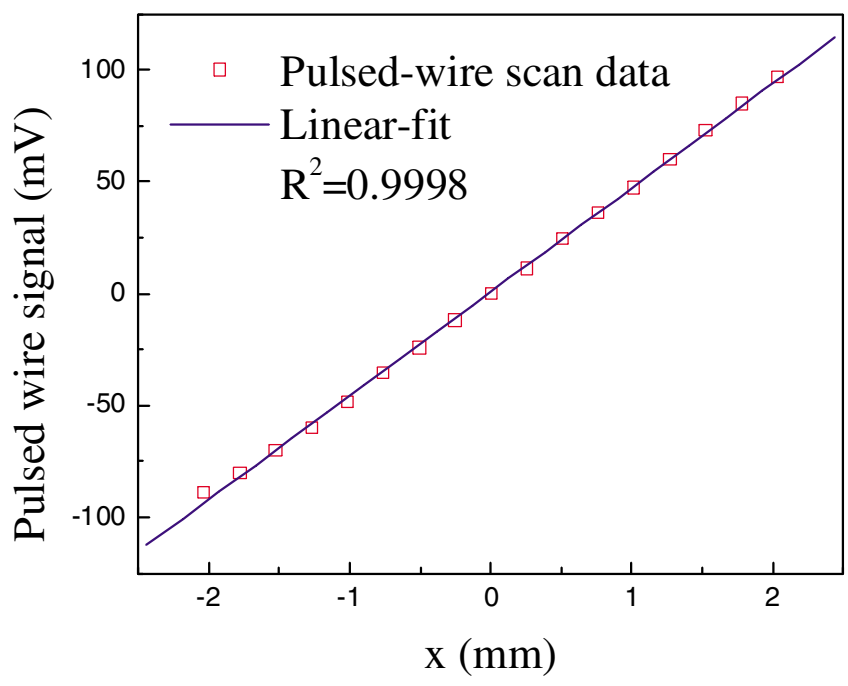

FIG. 16. (Color) Field linearity scan over accessible PMQ aperture from pulsed-wire measurement.

With the PMQ mounted on the same translation micrometer stages, the probe scans horizontally reading $B_{y}$. The field gradient obtained from this measurement in the center of the PMQ bore region was measured to be $560 \mathrm{~T} / \mathrm{m}$ (see Fig. 2) using this method. We note that a Hall probe is limited in spatial resolution, which in turn limits the possible characterization of nonlinear field components in a quadrupole magnet.

An effective magnetic length was also determined using a Hall probe by reading the value of $B_{y}$ at each point along a longitudinal line located $0.5 \mathrm{~mm}$ diagonally from the magnet axial axis. The measured effective magnetic length of $10.4 \mathrm{~mm}$, as shown in Fig. 2, shows a good agreement with the RADIA simulation. While the entire range of the magnet is not accessible to measurement, the reproduction of the RADIA results allows us to deduce the effective magnet lengths, which are employed as input in the beam dynamics simulations, to be essentially those given by RADIA.

\section{BEAM-BASED MEASUREMENTS}

\section{A. The PLEIADES experiment}

The PMQ system was installed in the PLEIADES ICS experiment, in which a relativistic $(25-80 \mathrm{MeV})$, highbrightness electron beam is collided with a $50 \mathrm{fs}$, up to $1 \mathrm{~J}$, $820 \mathrm{~nm}$ laser to produce short pulse $(0.3-5 \mathrm{psec})$, high peak brightness, hard $\mathrm{x}$ rays, tunable in energy from $40-$ $140 \mathrm{keV}$.

The number of x-ray photons, $N_{x}$, produced by the interaction of the laser and electron beams is determined by the intensities of the beams as they overlap,

$$
N_{x}=\sigma_{T} c(1-\vec{v} \cdot \vec{k}) \int_{V, t} n_{L}(\vec{x}, t) n_{e}(\vec{x}, t) d V d t,
$$


where $n_{L}(\vec{x}, t)$ and $n_{e}(\vec{x}, t)$ are the laser photon and electron beam densities, respectively, $\sigma_{T}$ is the total Thomson cross section, $\vec{v}$ is the electron beam velocity, and $\vec{k}$ is the laser propagation wave number. For the PLEIADES experiment, a $180^{\circ}$ scattering geometry is used. The integrated $\mathrm{x}$-ray flux is found, assuming Gaussian spatial and temporal profiles for both beams, and that the laser and electron beam pulse durations are less than the Rayleigh range and $\beta$ function of the laser and electron foci, respectively, to be

$$
N_{x}=\frac{N_{e} N_{L} \sigma_{T}}{2 \pi\left(\sigma_{e}^{2}+\sigma_{L}^{2}\right)} .
$$

Here $\sigma_{e}$ and $\sigma_{L}$ are the electron and laser beam spot sizes at the interaction point, and $N_{e}$ and $N_{L}$ are the total numbers of electrons and laser photons in the pulses. Note that PLEIADES experimental design parameters include a bunch charge approaching $1 \mathrm{nC}\left(N_{e}=6 \times 10^{9}\right)$ and a laser photon energy approaching $1 \mathrm{~J}$ at a wavelength around $1 \mu \mathrm{m}$, resulting in $N_{L}=5 \times 10^{18}$. Assuming the incident laser and electron beams are matched such that $\sigma_{e}=\sigma_{L}$, then to obtain a flux of $\mathrm{x}$ rays suitable for a single shot diffraction experiment (about $10^{8}$ ), an rms interaction spot size in the tens of microns is required.

Further, since initial x-ray data were obtained with $\sigma_{L}<$ $\sigma_{e}$, the PLEIADES ICS experiment benefits significantly from decreasing the electron beam size at the interaction point and is therefore an excellent location to initially implement the PMQ final-focus system.

\section{B. Installation at PLEIADES}

The PLEIADES electron beam is generated using a BNL/SLAC/UCLA style 1.6 cell photocathode rf gun [24]. Following the gun, the beam is accelerated to energies of $20-100 \mathrm{MeV}$ using as many as four $S$-band $2.5 \mathrm{~m}$ traveling wave sections. For the measurements described below, the gun produced $200-300$ pC bunches with 3 ps rms duration, while the final beam energy was varied between 50 to $70 \mathrm{MeV}$, and the typical normalized, rms emittance was around $10 \mathrm{~mm}$ mrad.

To install the PMQs as the $e$-beam final-focus system, the high energy electron beam line was redesigned both to provide approximate beam matching into the PMQs and to retain diagnostic capabilities. A schematic of this beam line is shown in Fig. 17.

The laser-electron IP (interaction point) is located at the center of a 6-inch cube vacuum chamber. As described above, the PMQ mover assembly is mounted directly into the interaction chamber, allowing positioning of the magnets at a range of distances directly upstream of the IP (see Fig. 18). The minimum distance of the final PMQ to the IP is determined by the width of a polished aluminum cube positioned at the IP. This cube functions as the spatial and temporal overlap diagnostic by directing the laser light and the optical transition radiation (OTR) produced by the electron beam into a CCD camera and a streak camera. In addition to enabling the overlap of the two beams, the CCD images naturally allow optimization of the focused spot size. This cube is extracted from the beam path for $\mathrm{x}$ ray production but cannot be fully removed from the PMQ path. As a result, the minimum distance from the edge of the final PMQ to the IP is $5 \mathrm{~mm}$.

After the IP, a round pole dipole magnet is used to bend the electron beam onto a trajectory which separates it from the x-ray pulse, allowing the beam to be dumped into a shielded Faraday cup, minimizing the noise reaching the $x-$ ray detectors. Note that a spectrometer magnet was also located just upstream of the final matching quads to measure the energy and energy spread of the beam, which are essential steps in tuning the linac.

\section{Tuning the final-focus optics}

In accordance with the quad triplet model used to design the PMQ final-focus system, the beam must be manipu-

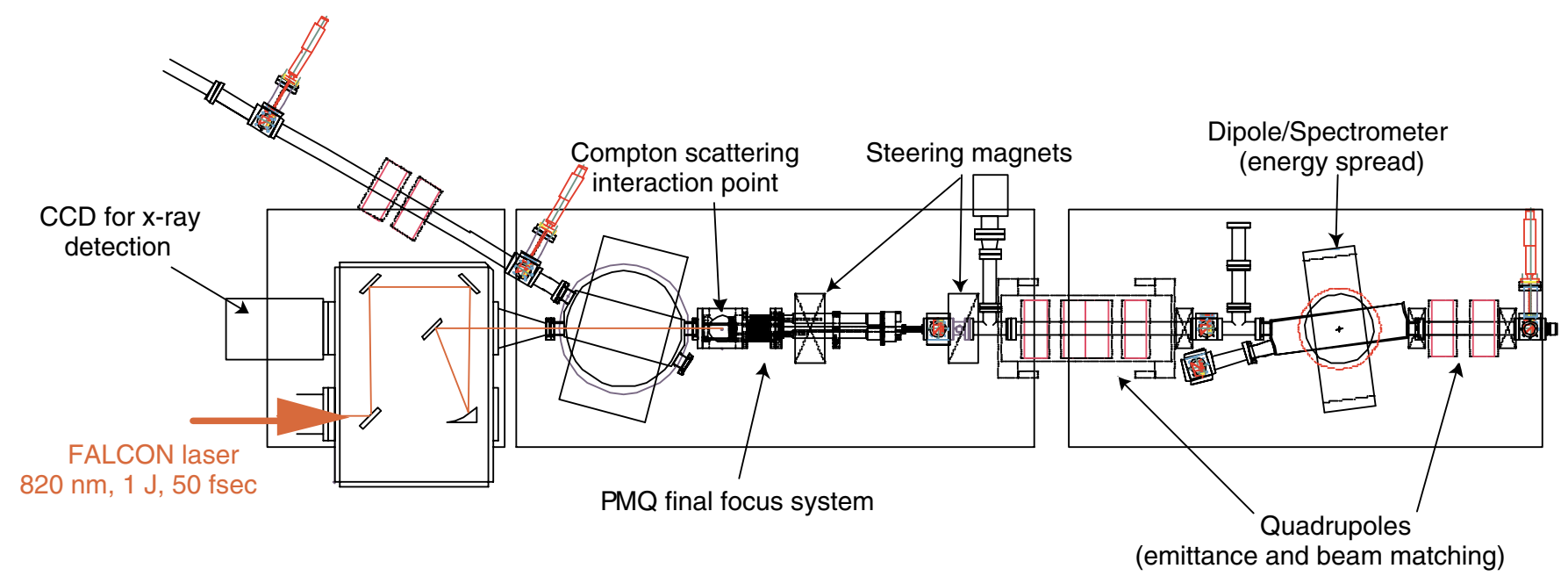

FIG. 17. (Color) Schematic drawing of the PLEIADES interaction beam line. 


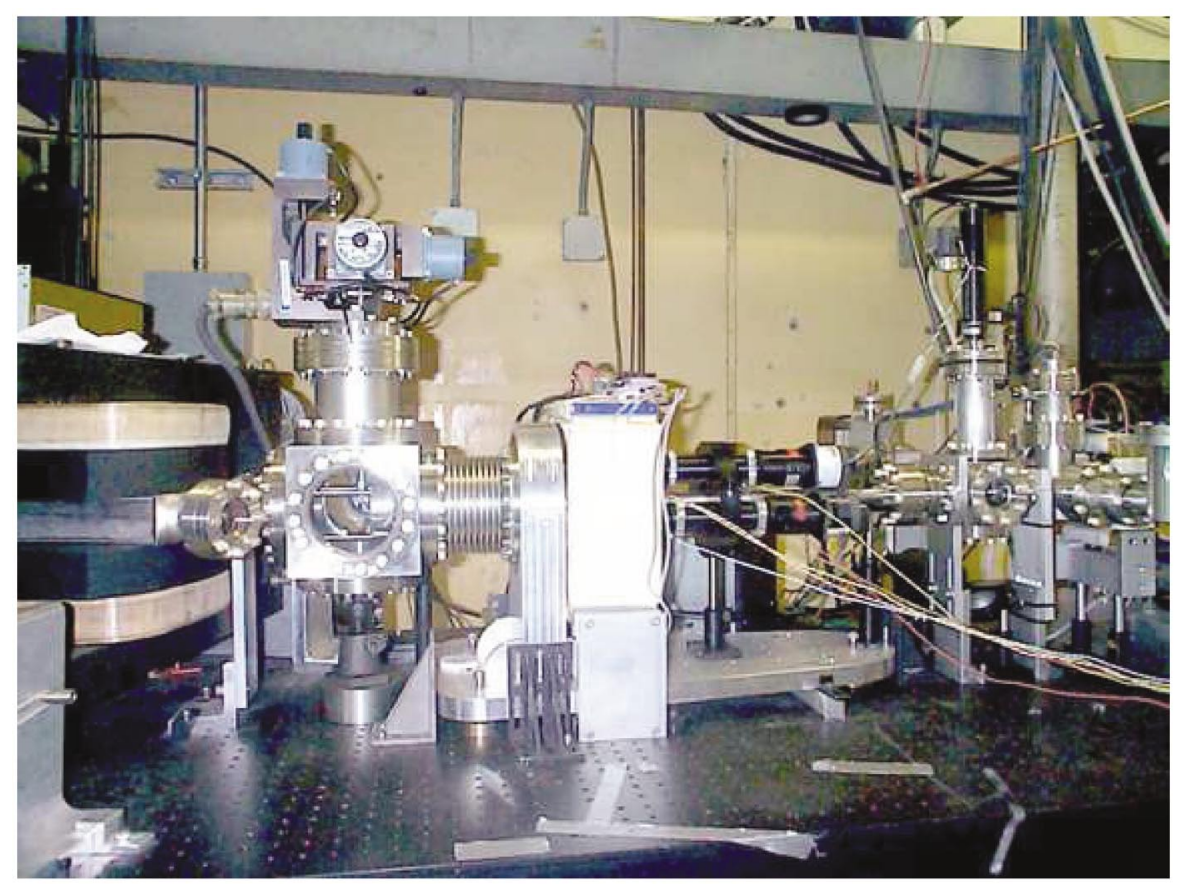

FIG. 18. (Color) The PMQ final-focus assembly after installation at PLEIADES. The vacuum cube enclosing the IP is shown on the left.

lated upstream of the PMQs to produce a moderately focused waist at the entrance to the first PMQ. The first step in this process is the determination of the rms Twiss parameters of the beam exiting the accelerator, which is done using the quadrupole scan emittance measurement technique. In the quad scan measurement, the current in the first electromagnetic (EM) quad in Fig. 17 is varied while the rms beam size is measured after a known drift length. Taking into account the finite length of the quadrupole magnet (using a "thick lens" analysis) the final beam size in each transverse direction, $\sigma_{f}$, follows the wellknown relation [25]

$$
\begin{aligned}
\sigma_{f}^{2}= & {\left[\cos \left(\sqrt{K} l_{q}\right)-\sqrt{K} l_{q} \sin \left(\sqrt{K} l_{q}\right)\right]^{2} \sigma_{11, i} } \\
& +2\left[\frac{\sin \left(\sqrt{K} l_{q}\right)}{\sqrt{K}}+l_{d} \cos \left(\sqrt{K} l_{q}\right)\right]\left[\cos \left(\sqrt{K} l_{q}\right)\right. \\
& \left.-\sqrt{K} l_{d} \sin \left(\sqrt{K} l_{q}\right)\right] \sigma_{12, i}+\left[\frac{\sin \left(\sqrt{K} l_{q}\right)}{\sqrt{K}}\right. \\
& \left.+l_{d} \cos \left(\sqrt{K} l_{q}\right)\right]^{2} \sigma_{22, i}
\end{aligned}
$$

where $l_{q, d}$ are the EM quad and drift lengths, respectively, $K$ is the focusing strength of the quad given by its magnetic field gradient and the beam momentum, $K=e B^{\prime} / p$, and the initial Twiss parameters are $\beta_{i}=\sigma_{11, i} / \varepsilon, \alpha_{i}=$ $-\sigma_{12, i} / \varepsilon$, and $\gamma_{i}=\sigma_{22, i} / \varepsilon$, where $\varepsilon$ is the rms emittance. Because quad scans must be performed as part of the routine operating procedure, the process was automated using LabVIEW based control system software. The im- mediate determination of the emittance provides the linac operator with feedback on the quality of the linac tune, as well as giving the Twiss parameters needed for matching the beam into the PMQs.

Once the Twiss parameters have been determined, they are used as input to a TRACE3D model of the beam line. Given the beam parameters at the first element of the model, and those desired at some latter point, TRACE3D can perform a matching function, in which a user defined set of model parameters is adjusted in an algorithm to produce the desired final beam. In this case, we adjust the strengths of EM quad doublet and triplet to produce a beam waist $(\alpha=0)$ in both transverse dimensions at the entrance to the PMQs with an rms size around $350 \mu \mathrm{m}$. An example of the output of this matching process is shown in Fig. 19. The choice of rms beam size at the final-focus entrance is a compromise between emittance and aberration considerations, and will be discussed further below.

Once the TRACE3D model has determined the proper settings for the upstream optics, measuring the beam size at two pop-in beam profile monitors and comparing the results with the model predictions allows a partial check of the model. With good agreement found between model and measurement, TRACE3D is used again to determine the proper spacing of the PMQs. The result of this matching process is shown in Fig. 20. Here the spacing between 3 PMQ holders is adjusted to produce the smallest $\beta$ function possible at the IP.

The linac operator uses the TRACE3D values of upstream quad strengths and PMQ positions as a starting point. One important practical consideration the operator must take 


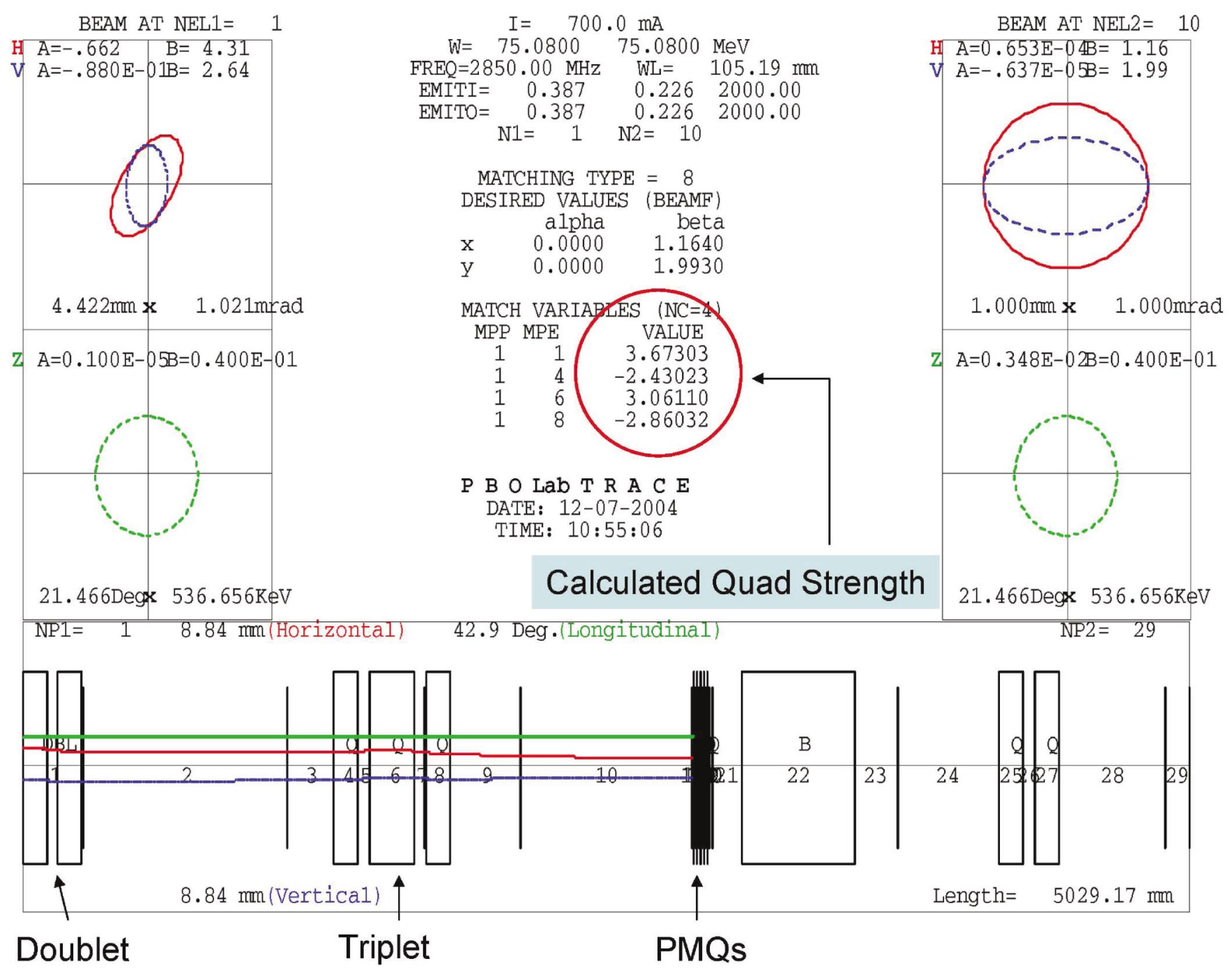

FIG. 19. (Color) TRACE3D (screen shot) model of the beam line used to compute the focusing strength of the upstream EM quads for proper matching into the PMQs.

into account is steering the beam onto the axis defined by the PMQ mover system. This is needed both to minimize the effects of chromatic and spherical aberrations in the PMQs and to precisely define the direction of the ICS $\mathrm{X}$ rays generated. There are two steering magnets located between the EM quad triplet and the PMQs that are used for this job. Steering is also made easier by imaging the beam on a plastic scintillator mounted on the upstream face of the first PMQ holder. This scintillator has a $5 \mathrm{~mm}$ diameter hole that is mounted concentric to the PMQ aperture. With these tools, proper beam steering is accomplished using the two steering magnets to iteratively move the beam to the center of the scintillator hole and observe the motion (or lack of motion) of the beam at the IP while moving the final PMQ holder.

The process described above has been used to focus the electron beam at the PLEIADES IP using the PMQ system. The spot size was measured using OTR generated by the beam colliding with the interaction cube diagnostic as described above. This light is collected by a CCD camera/lens system and digitized using a computer frame grabber. A typical and highly repeatable beam spot is shown in Fig. 21. In this case we focused a $70 \mathrm{MeV}$, $200 \mathrm{pC}$ beam to less than $20 \mu \mathrm{m}$ rms size in both transverse dimensions. This is a significant improvement over the previous final-focus system, which achieved spot sizes of roughly $50 \mu \mathrm{m}$, rms.

\section{Emittance measurements with the PMQs}

A variation of the quad scan technique for emittance measurement is the so-called three screen or moving screen measurement, in which there is a focusing lens followed by a drift space and measurement screen. However, in this case the beam size is measured while varying the drift length instead of the lens strength. In the simplified thin-lens analysis (where the focal length is $f=$ $1 / K l_{q}$ ) the equation analogous to Eq. (13) is 


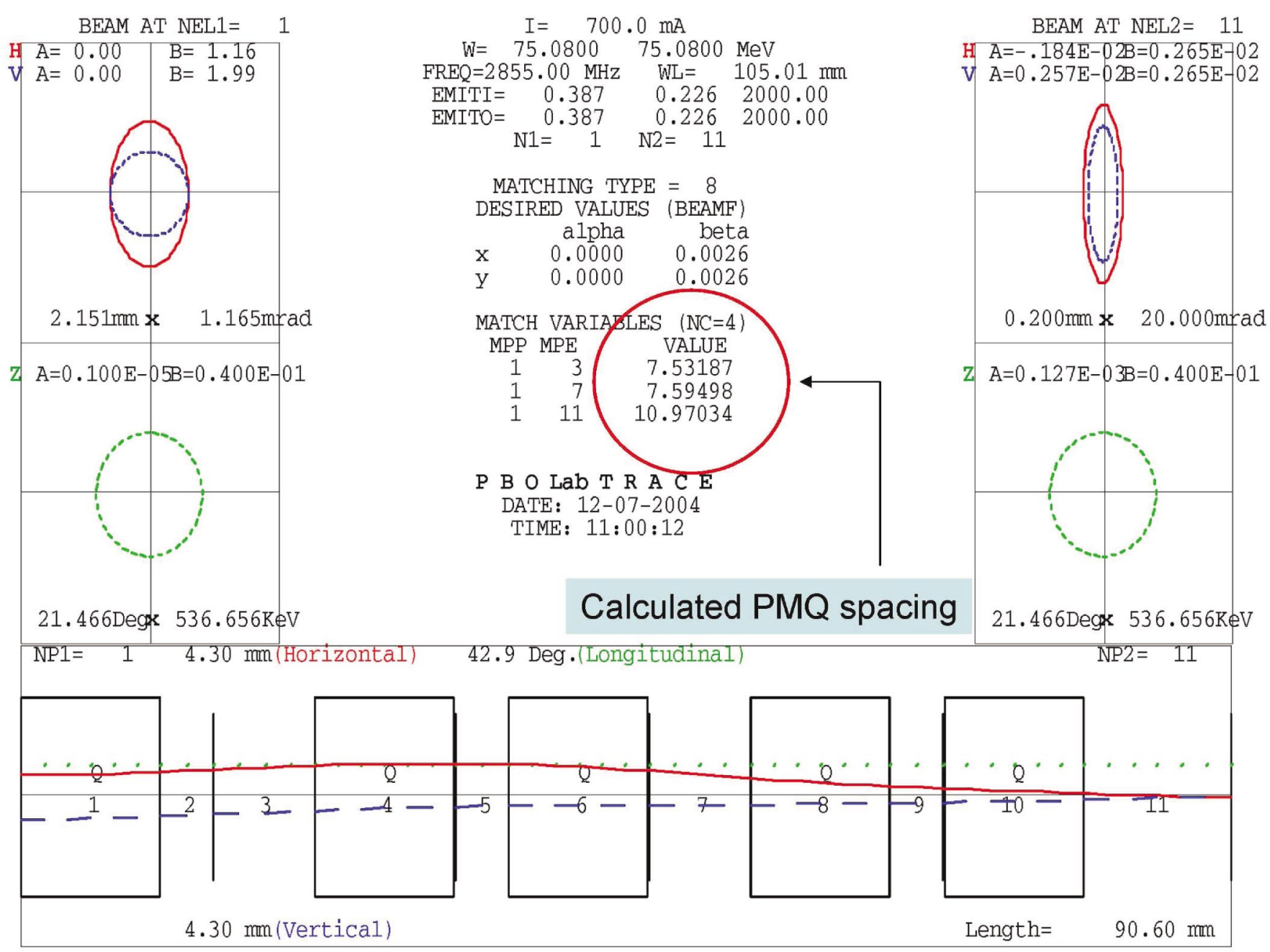

FIG. 20. (Color) Typical TRACE3D output (screen shot) specifying the spacing between PMQs and their position with respect to the beam focus.

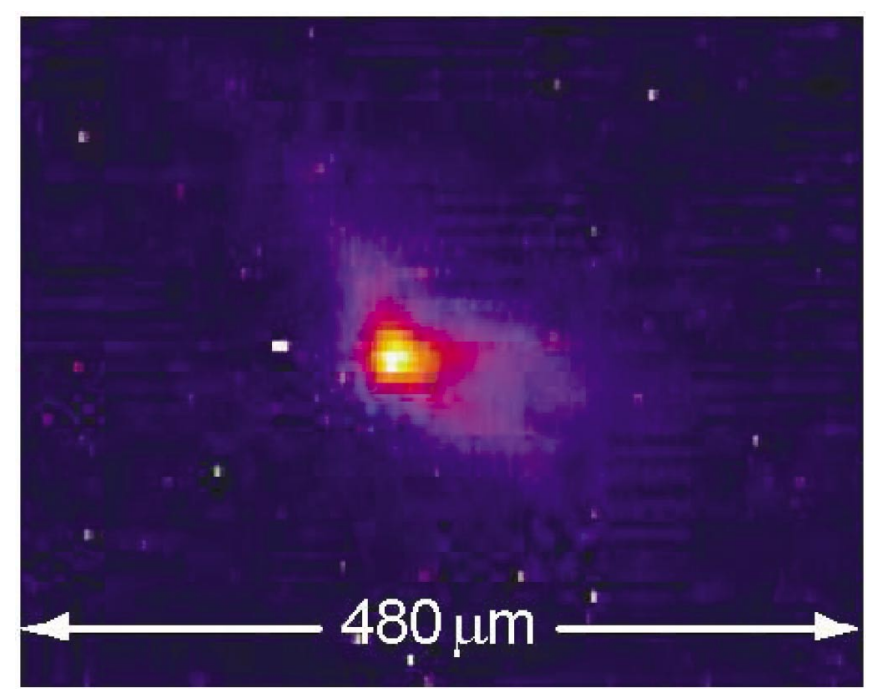

FIG. 21. (Color) False color CCD image of the PMQ focused electron beam. The rms beam size is $20 \mu \mathrm{m}$ in both $x$ and $y$.

$$
\sigma^{2}(z)=\beta^{*} \varepsilon_{0}\left[1+\left(\frac{z-z_{0}}{\beta^{*}}\right)^{2}\right]+\sigma_{\text {res }}^{2},
$$

where $\beta^{*}$ is the minimum $\beta$ function at the waist, $\varepsilon_{0}$ is the beam emittance, and $\sigma_{\text {res }}$ is the image resolution obtained from the limitations of optical transport and camera performance. In the free drift, the square of the rms beam spot size expands quadratically away from the beam waist position, $z_{0}$.

In the case of the PMQ final-focus system, the focal length, $f$, is fixed and the drift length, $z$, is changed most easily by moving all three of the PMQ holders in unison. This is not technically a correct application of Eq. (14), since by moving the quadrupoles instead of the screen, we are varying the initial Twiss parameters. However, since we know the initial $\beta$ function of the input beam to be at least $1 \mathrm{~m}$, and since the scans must only cover several $\beta^{*}$, or less than $1 \mathrm{~cm}$, the initial conditions remain essentially constant over the scan. 
Thus, the analysis of the moving screen technique to the "moving quads" measurement is valid in the present scenario. One such dataset and analysis is shown in Fig. 22. The effects of limited image resolution can be clearly seen in these measurements. The expected parabolic dependence of $\sigma^{2}$ on $z$ is observed well away from the minimum beam sizes, but the data give a nearly flat dependence in the zone near $z_{0}$. In the case studied in Fig. 22, normalized initial emittances measured at $58.2 \mathrm{MeV}$ beam energy using the EM quad triplet are $\varepsilon_{x}=16.6 \mathrm{~mm}$ mrad and $\varepsilon_{y}=9.7 \mathrm{~mm}$ mrad, while the normalized emittances obtained with the PMQ scan are $\varepsilon_{x}=16.3 \mathrm{~mm}$ mrad and $\varepsilon_{y}=12.9 \mathrm{~mm} \mathrm{mrad}$. During the initial period of running the final-focus system, beam spot sizes as low as $\sigma_{\text {rms }} \sim$ $18 \mu \mathrm{m}$ were measured in both transverse dimensions after optimization.

As noted, this is an upper limit on the actual beam size, due to the resolution limitations in the measurement system. We note from Fig. 22 that the value of the $\sigma_{\text {res }}$ obtained from the best fit of the quad scan data to Eq. (14) is also $18 \mu \mathrm{m}$. With the value of $\sigma_{\text {res }}$ determined by the fit to the data, we deduce that the minimum beam size in each transverse dimension in Fig. 22 would have been in the range of $15 \mu \mathrm{m}$ in the absence of image resolution problems (e.g., geometric and chromatic aberrations). In order to observe such small sizes, a reconfiguring of the optics between the interaction region OTR surface and the viewing CCD camera will be undertaken in the future. One of the most critical needed capabilities the PMQ-based final-focus system, that of tunability over a wide range of beam energies, has been demonstrated.
Through longitudinal motion of the PMQs, the final-focus system was capable of adjusting to consistently produce a small beam spot size at different input beam energies. This variability, over $40 \%$ in beam energy, has produced variation of $\mathrm{x}$-ray photon energies over the range between 40 to $140 \mathrm{keV}$.

Recently, the final-focus system was successfully reconfigured by boring out the initial isolated PMQ's inner diameter to lower its gradient, and removing one of each of the downstream PMQ pairs. This new system has allowed production of an optimum beam focus at as low as $20 \mathrm{MeV}$ electron beam energy. This new capability has allowed use of PLEIADES to pursue ongoing dynamic pump-probe experiments which investigate dynamic diffraction in different heavy-metal materials.

\section{DISCUSSIONS AND CONCLUSIONS}

An adjustable short focal length final-focus system based on an asymmetric (F-DD-FF) triplet configuration composed of unprecedented ultrahigh field gradient, with experimental value $\sim 560 \mathrm{~T} / \mathrm{m}$, PMQs has been developed. The design issues concerning the PMQs themselves were studied in detail using the 3D magnetostatic simulation code RADIA. The PMQ array was fabricated, measured, and placed on a precision moving system. It was then implemented at the ICS final-focus system beam line at LLNL PLEIADES. This effort led to creation of ultrasmall beam spot sizes, and an enhanced performance of a highbrightness ICS x-ray source, with adjustability demonstrated over a wide range of input beam energies, and thus produced $\mathrm{x}$-ray energies.

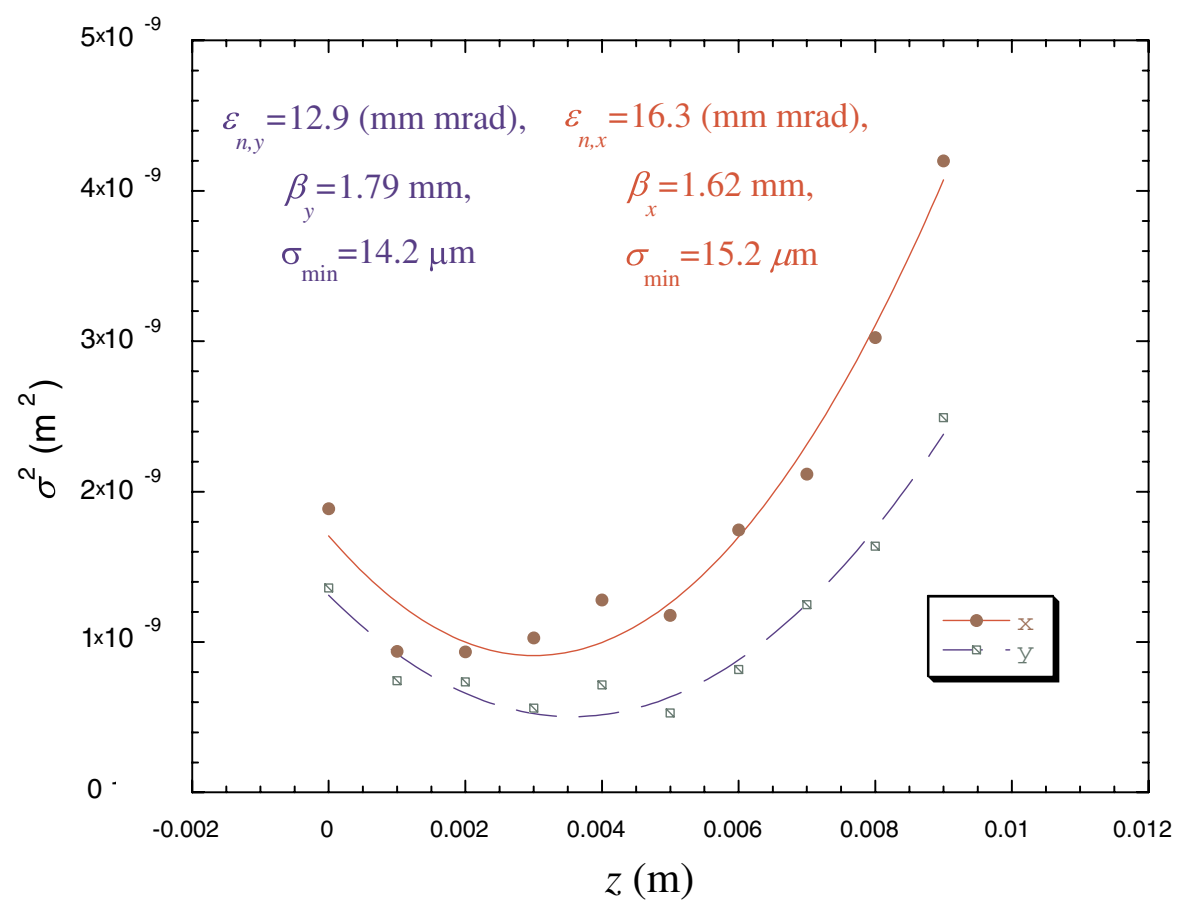

FIG. 22. (Color) Quadrupole scan of the final-focus beam at LLNL PLEIADES, performed by moving the entire PMQ assembly. 
The principles behind the short focal length, adjustable PMQ-based final-focus system are quite attractive for advanced applications. At UCLA, a similar system, with few $\mathrm{cm}$ focal lengths, is presently being implemented in the final focus of a nonlinear ICS experiment in the Neptune laboratory. As the emittance is expected to be a factor of 3 lower in the Neptune experiment, this test should allow demonstration of the emittance preservation at a level much closer to the state-of-the-art in high-brightness photoinjector systems. Additionally, the current experience in creating very high gradient, small-bore quadrupoles is of continuing interest for application to linear collider finalfocus systems, as the demonstrated gradient achieved here exceeded the CLIC efforts by over $15 \%$. Finally, we note that standard F-DD-F PMQ triplets using longitudinal position tuning, with much larger bore and lower gradients have been used at LANSCE for proton radiographic microscopy [26], an application which does not require large demagnification, but nonetheless demands very high strength quadrupoles.

\section{ACKNOWLEDGMENTS}

The authors would like to thank G. Andonian and J. England for their assistance with simulations. The authors also thank G. Anderson for the assistance in fielding experiments. This work was performed under the auspices of the U.S. Department of Energy under Contracts No. DEFG-98ER45693 and No. DE-FG03-92ER40693, and by Lawrence Livermore National Laboratory under Contract No. W-7405-Eng-48 and the LLNL ILSA program under Contract No. LS04-001-B.

[1] J. S. Fraser, R. L. Sheffield, E. R. Gray, and G. Rodenz, in IEEE Trans. Nucl. Sci. 32, 1791 (1985).

[2] P. Chen, J. M. Dawson, R. W. Huff, and T. Katsouleas, Phys. Rev. Lett. 54, 693 (1985).

[3] J. B. Rosenzweig, B. Breizman, T. Katsouleas, and J. J. Su, Phys. Rev. A 44, R6189 (1991).

[4] R. W. Schoenlein et al., Science 274, 236 (1996).

[5] I. V. Pogorelsky et al., Phys. Rev. ST Accel. Beams 3, 090702 (2000).

[6] L. Serafini and J. B. Rosenzweig, Phys. Rev. E 55, 7565 (1997).
[7] J. B. Rosenzweig and P. Chen, Phys. Rev. D 39, 2039 (1989).

[8] S. Anderson et al., Appl. Phys. B 78, 891 (2004).

[9] D. J. Gibson et al., Phys. Plasmas 11, 2857 (2004).

[10] W. Lou, D. Hartill, D. Rice, D. Rubin, and J. Welch, in Proceedings of the Particle Accelerator Conference, Vancouver, BC, Canada, 1997 (IEEE, Piscataway, NJ, 1998), Vol. 3, p. 3236.

[11] J. T. Volk et al., in Proceedings of the Particle Accelerator Conference, Chicago, IL, 2001 (IEEE, Piscataway, NJ, 2001), Vol. 1, p. 217.

[12] F. Zimmermann, R. Assmann, G. Guignard, D. Schulte, and O. Napoly, in Proceedings of the European Particle Accelerator Conference, Vienna, 2000 (EPS, Geneva, 2000).

[13] F. Zimmermann, R. Assmann, G. Guignard, D. Schulte, and O. Napoly, CERN Technical Report No. CERN-SL2000-057 AP, 2000.

[14] S.C. Gottschalk, W.D. Kimura, and D. H. Dowell, in Proceedings of the Particle Accelerator Conference, Chicago, IL, 2001 (Ref. [11]), Vol. 5, p. 3218.

[15] R. B. Brown and J. R. Cost, IEEE Trans. Magn. 25, 3117 (1989).

[16] K. Halbach, Nucl. Instrum. Methods 169, 1 (1980).

[17] W. Lou, D. Hartill, D. Rice, D. Rubin, and J. Welch, Phys. Rev. ST Accel. Beams 1, 022401 (1998).

[18] P. Elleaume, O. Chubar, and J. Chavanne, in Proceedings of the Particle Accelerator Conference, Vancouver, BC, Canada, 1997 (Ref. [10]), Vol. 3, p. 3509.

[19] J. Chavanne, O. Chubar, P. Elleaume, and P. V. Vaerenbergh, in Proceedings of the European Particle Accelerator Conference, Vienna, 2000 (Ref. [12]), p. 2316.

[20] K. Crandall and D. Rusthoi, Los Alamos National Laboratory Technical Report No. LA-UR-97-886, 1997.

[21] M. Borland, Advanced Photon Source Technical Report No. LS-287, 2000.

[22] A. Chao and M. Tigner, Handbook of Acccelerator Physics and Engineering (World Scientific, Singapore, 1999).

[23] R. Warren, Nucl. Instrum. Methods Phys. Res., Sect. A 272, 257 (1988).

[24] D. T. Palmer, S. Anderson, and J.B. Rosenzweig, in Physics of High Brightness Beams (World Scientific, Singapore, 2000), p. 439.

[25] J. B. Rosenzweig, Fundamentals of Beam Physics (Oxford University Press, Inc., New York, 2003).

[26] T. Mottershead et al., in Proceedings of the Particle Accelerator Conference, Portland, OR, 2003 (IEEE, Piscataway, NJ, 2003), Vol. 1, p. 702. 